\title{
Design Genetic Algorithm To Find The Optimal Critical Path Network Project (GAOCPN)
}

Samaa Tlayea Azeez

Niam Al-Thanoon

Lamyaa Jasim Mohammed samatalee843@gmail.com niam.munim@uomosul.edu.iq Lomuaa Jasem@uomosul.edu.iq College of Computer Sciences and Mathematics

University of Mosul

\section{Received on: 30/6/2011}

\section{ABSTRACT}

Accepted on:2/11/2011

The present study deals with using up-to-date intelligent techniques. We try to utilize the genetic algorithm efficiently and integrate it with the problem of study by designing and applying a genetic algorithm to find the optimal critical path of networks GAOCPN achieving many results, e.g., real time. Accuracy in representing the steps of project execution as a net of nodes and paths has a great role in the accuracy of program results GAOCPN written in C++ version 5.0 under Window. The program was applied on many networks, such as Al-Sarafiya Bridge networks, and the execution time and results were checked and compared with the execution time and results of traditional methods (dynamic programming) and Win_QSB program. The GAOCPN showed accuracy of results in a standard time. Sometimes, it showed optimal results better than those of the traditional methods and it showed results identical to Win_QSB but in standard time.

Keywords: genetic algorithm, critical path, dynamic programming, project network.

تصميم خوارزمية جينية لإيجاد المسار الحرج الأمثل لثبكة أعمال المشاريع (GAOCPN)

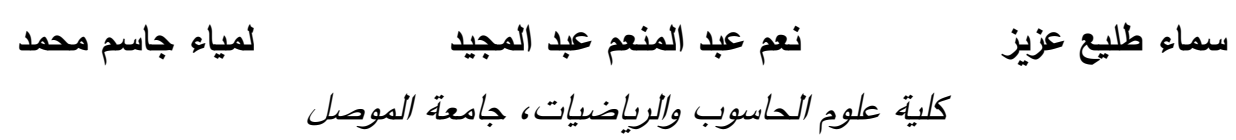

$$
\text { تاريخ قبول البحث: 2011/11/02 }
$$

تاريخ استلام البحث: 2011/06/30

\section{الملخص}

تركز الاهتمام في هذا البحث على استخدام التقنيات الذكائية المعاصـرة إذ نحاول توظيف الخوارزمية

الجينية بثكل كفوء وسلس ومتكامل مع طبيعة مشكلة البحث وذلك بتصميم وتطبيق خوارزمية جينية لإيجاد المسار الحرج الأمثل لشبكات الأعمال GAOCPN محاولين بذلك تحقيق عدة مسائل منها مسألة الوقت الحقيقي. إن الدقة في تمثيل خطوات انجاز المشروع بوصفه شبكة من العقد والمسارات له الدور البارز والحازم في دقة نتائج البرنامج GAOCPN الذي كتب بلغة C++ الإصدار الخامس ببيئة Window. طبق البرنامج على عدة شبكات أعمال ومنها شبكة أعمال جسر الصرافية وتم التأكد من نتائج ووقت التنفيذ ومقارنتهما مع نتائج ووقت التنفيذ لكل لئل من الطرائق التقليدية (البرمجة الديناميكية) والبرنامج الجاهز Win_QSB. وأثبت GAOCPN دقة النتائج وبوقت

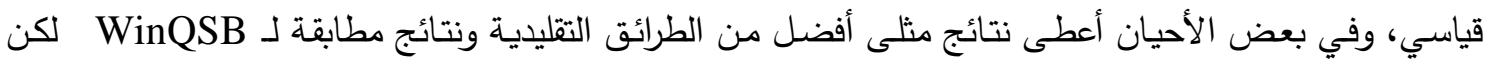
بوقت قياسي.

الكلمات المفتاحية : الخوارزمية الجينية ، المسار الحرج , البرمجة الديناميكية , مشروع شبكات الاعمال.

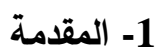

إن البرمجة الديناميكية المتمثلة بالطريقة الحسابية المباشرة وطريقة البرمجة الخطية بخوارزميتيها الأمامية

والعكسية تعد من الطرائق التقليديـة في بحوث العمليات والتي استخدمت لإيجاد المسار الحرج الأمثل لانجـاز

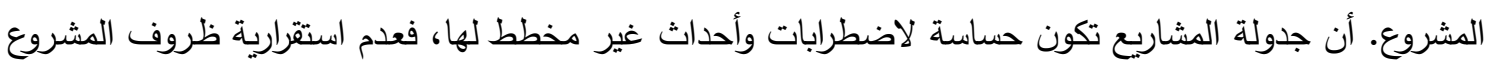
(اللاتأكدية) التي تحدث فجأة في بيئة المشروع تتطلب إعادة صدياغة الثبكة (استحداث/حذف /تغيير قيمة نشاط أو تباطؤ تتفيذ نشاط) ويطلق عليها التوسع التقدمي الذي يتسبب في بذل وقت وجهد أضافي لإعادة تلك الحسابات 
المعقدة. وبالتالي فان أحداث الوقت الحقيقي سيؤثر على هيكل الثبكة وعلى كلفة ووقت أنجاز المشروع، وعليه

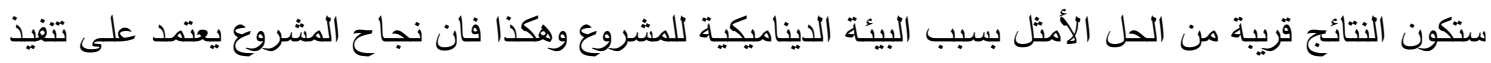

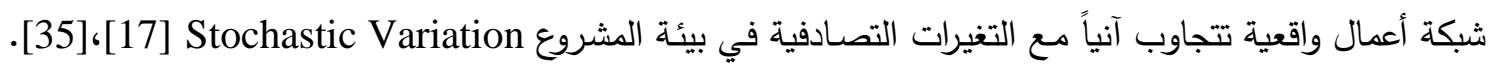
تم تصميم GAOCPN بوصفه تقنية ذكائية لإيجاد المسار الحرج الأمثل بدون الحاجة إلى دراسة طرائق بحوث

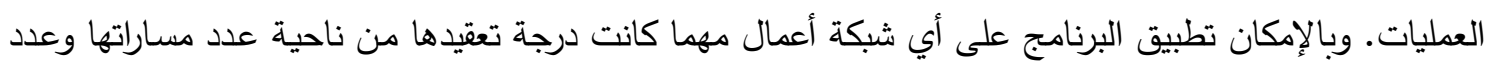
عقدها وعدد مراحلها بسلاسة ومرونة [4]، [24].

\section{2- المفاهيم الأساسية لتمثيل نماذج مخطط شبكة المشروع}

كل مشروع يتكون من عدة أنشطة وتتجز الأنشطة تباعا حسب تسلسلها الذي يؤخذ من أدارة الموارد.

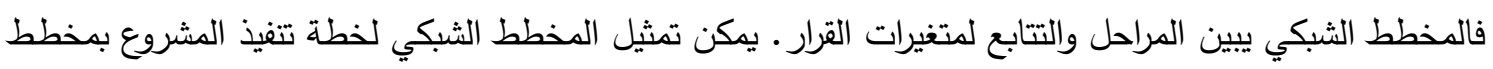

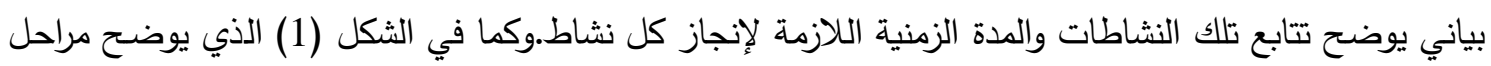

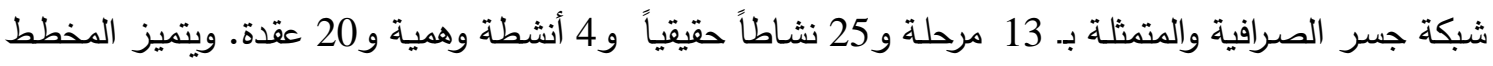

$$
\text { الشبكي بالاتي [6]،:[13]: }
$$

أ. النشاط Activity: يعبر عنه بسهح يربط بين عقدتين, حيث يتطلب تتفيذ النشاط موارد بشرية ومادية ووقت,

لاحظ الجدول (1).

ب. الحدث Event: يعبر عنه بعقدة وتظهر عند بداية ونهاية كل نشاط, وهو لحظة من الزمن لا يتطلب موارد ووقتاً.

ج. النشاط الوهمي Dummy Activity: يعبر عنه بسهم متقطع وزمن تتفيذها صفراً ولا تستفذ أي موارد، ويضاف إلى المخطط الثبكي لضمان صحة التسلسل والترابط المنطقي.

د. لا يجوز تكرار العقدة نفسها بالثبكة وبالتالي فان المسارات الحرجة لا تتكرر فيها أي عقدة.

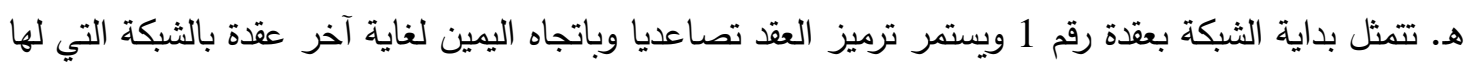
اكبر رقم.

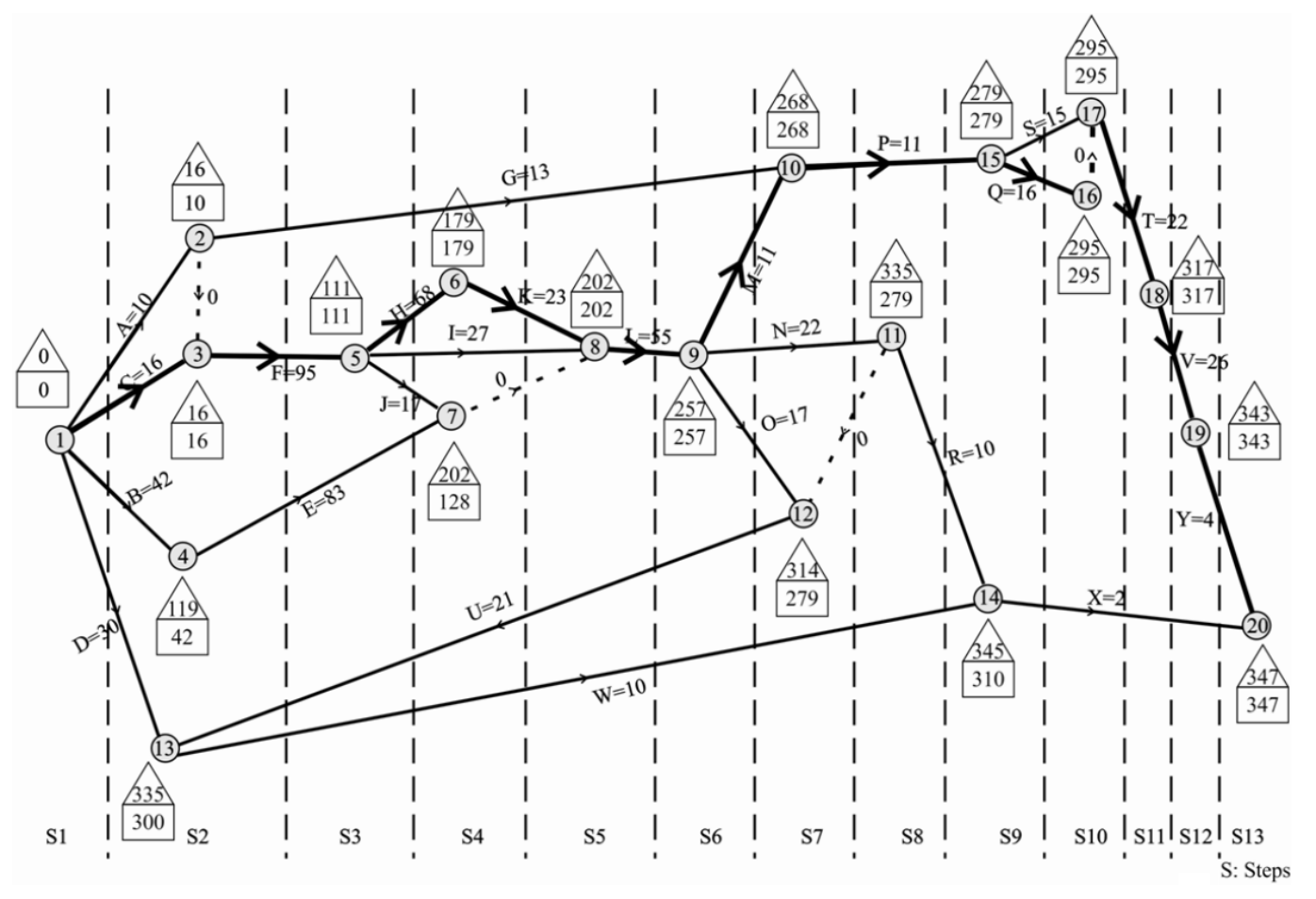

الثكل (1). شبكة أعمال جسر الصر افية ممثلة8هاليخط الشبكي السهمي AOA من مصدر [1] 
(S1, S2, ., , S13)

الأوقات المبكرة والمتأخرة حسبت من قبل الباحثين.

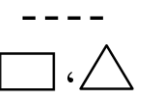

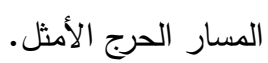

الجدول (1). تعاقب أنشطة شبكة أعمال جسر الصرافية والأوقات المتوقعة لها من مصدر [1]

\begin{tabular}{|c|c|c|c|c|c|c|c|}
\hline Et & $\mathbf{a}$ & $\mathbf{m}$ & b & وصف النشاط & النشاط السابق & رمز النشاط & $ت$ \\
\hline 10 & 7 & 10 & 15 & أعمال التسوية والدفن بالتراب & - & A & 1 \\
\hline 42 & 30 & 40 & 60 & توفير وشراء المواد الأولية & - & B & 2 \\
\hline 16 & 10 & 15 & 25 & تقيئة ونصب البانتونات & - & $\mathrm{C}$ & 3 \\
\hline 30 & 20 & 30 & 40 & نصب الجسر العائم & - & $\mathrm{D}$ & 4 \\
\hline 83 & 60 & 80 & 120 & تصنيع الهيكل الحديدي & B & $\mathrm{E}$ & 5 \\
\hline 95 & 75 & 90 & 135 & تفكيك ورفع الغوارق & $\mathrm{C}, \mathrm{A}$ & $\mathrm{F}$ & 6 \\
\hline 13 & 10 & 12 & 20 & قلع المتبقي من التبليط السابق & A & G & 7 \\
\hline 68 & 40 & 60 & 125 & معالجة الدعامات المتضررة & $\mathrm{F}$ & $\mathrm{H}$ & 8 \\
\hline 27 & 20 & 25 & 40 & دق الركائز الأنبوبية & $\mathrm{F}$ & I & 9 \\
\hline 17 & 10 & 15 & 30 & تثبيت وسادة حديدية بين الدعامات & $\mathrm{F}$ & $\mathrm{J}$ & 10 \\
\hline 23 & 15 & 20 & 40 & تجهيز وتثبيت مساند الارتكاز & $\mathrm{H}$ & K & 11 \\
\hline 55 & 40 & 50 & 90 & نصب الهيكل الحديدي & E, I, J, K & $\mathrm{L}$ & 12 \\
\hline 11 & 7 & 10 & 20 & تثبيت مفاصل التمدد & $\mathrm{L}$ & M & 13 \\
\hline 22 & 10 & 20 & 40 & قلع وإعادة تركيب أنابيب المياه & $\mathrm{L}$ & $\mathrm{N}$ & 14 \\
\hline 17 & 10 & 15 & 30 & رفع الركائز الأنبوبية & $\mathrm{L}$ & $\mathrm{O}$ & 15 \\
\hline 11 & 7 & 10 & 20 & صب سطح الجسر الخرساني & $\mathrm{G}, \mathrm{M}$ & $\mathrm{P}$ & 16 \\
\hline 16 & 10 & 15 & 25 & إجراء عملية العصف والصبغ الأولي & $\mathrm{P}$ & Q & 17 \\
\hline 10 & 7 & 10 & 15 & صيانة الأرصفة ومقتربات الجسر & $\mathrm{N}$ & $\mathrm{R}$ & 18 \\
\hline 15 & 7 & 14 & 25 & تبليط الجسر بالإسفلت ولعموم الجسر & $\mathrm{P}$ & $S$ & 19 \\
\hline 22 & 15 & 20 & 35 & صبغ الجسر بألاييوكسي & S, Q & $\mathrm{T}$ & 20 \\
\hline 21 & 15 & 20 & 30 & رفع البانتونات & $\mathrm{O}, \mathrm{N}$ & $\mathrm{U}$ & 21 \\
\hline 26 & 15 & 25 & 40 & الأعمال الكهربائية والخدمات & $\mathrm{T}$ & V & 22 \\
\hline 10 & 7 & 9 & 14 & رفع اللسان الترابي & $\mathrm{U}, \mathrm{D}$ & W & 23 \\
\hline 2 & 1 & 2 & 3 & صبغ الشارع وتخطيط المرور & $\mathrm{W}, \mathrm{R}$ & $X$ & 24 \\
\hline 4 & 3 & 4 & 6 & تنظيف المكان وتسليم المشروع & $\mathrm{X}, \mathrm{V}$ & $\mathrm{Y}$ & 25 \\
\hline
\end{tabular}

إذ أن الأوقات a و b و m يتم تخمينها من قبل الخبراء.

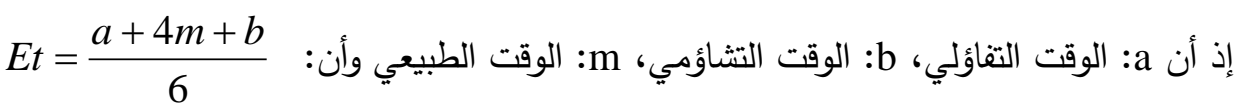

ويمكن تمثيل المخطط الثبكي بصيغتين هما [6]، [36] :

\section{1- المخطط الشبكي السهمي Activity on Arrow (AOA)}

يعد أكثر شيوعاً، إذ يوضع احد المعايير الوقت، الكلفة، الربح على النشاط. وقد نستعين بنشاط وهمي أو أكثر في هذا النوع من الثبكات. 


\section{2- المخطط الشبكي العقدي Activity on Node (AON)}

يوضح معيار الوقت،الكلفة،الربح في العقدة. في هذه الطريقة لا توجد أنشطة وهميـة, ولتوضيح حالات تمثيل

الأنشطة بين صيغتي AON و AOA لاحظ الثكل (2). الثكل (2). الفرق بين صيغتي AOA و AON

(AON) شبs) (AOA)

3- تصنيف مسائل الأمثلية

إن جذور الأمثلية المعاصرة يعود إلى الحرب العالمية الثانية وهو مولد بحوث العمليات بتطبيق الطرائق

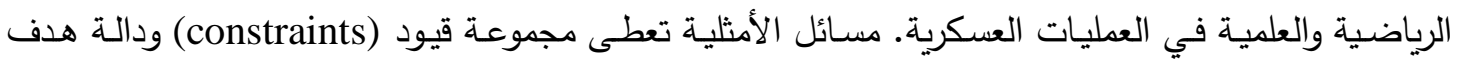
مجموعة الحلول التي تحقق القيود تسمى حلولا مكنة (objective function)

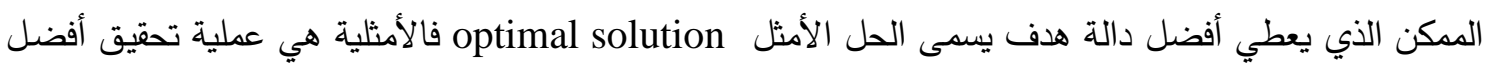
حل أو نتائج للأهداف المحددة من بـين مجموعـة من الحلول البديلـة بدون الاضطرار إلى تجريب كل البدائل

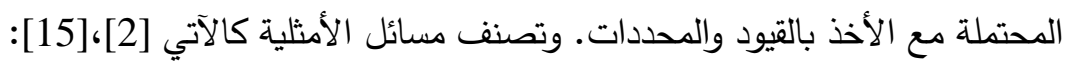
1- بواسطة الأنموذج By model يمثل إمـا بمتغير أو عدة متغيـرات, بقيود أو عدمها, كالمسيائل الخطيـة

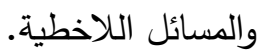

2- بواسطة طريقة الحل By Solution Method: كالبرمجة الديناميكية,البرمجة التربيعية التتابعية، البرمجة

$$
\text { الصحيحة، البحث الذهبي، البرمجة الهندسية. }
$$

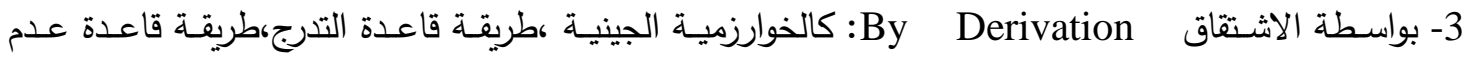

$$
\text { التدرج(العشوائي والبسيط). }
$$

4- حسب طبيعة المعلومات By Nature of Information: النماذج الاحتمالية والمحددة.

1-3 مراحل تطور أساليب الأمثلية التقليدية في المخططات الثبكية للمشاريع: نظرياً وعملياً

تطورت أسـاليب تتفيذ المشاريع ونتيجـة لذلك تعددت القواعد الاستكثـافية للجدولة سواء التقليدية (بحوث

$$
\text { العمليات) والذكائية (الخوارزمية الجينية) وكما يلي: }
$$


Gantt 1-1-3 مخطط

خريطة الجدول الزمني منسوبة إلى Henery Gantt والذي ابتدعها في عام 1917 ومازالت مستخدمة حتى الآن بل هي أشهر وسيلة مستخدمة في عرض الجداول الزمنية. وهي عبارة عن رَسم بياني يوضـح الجدول

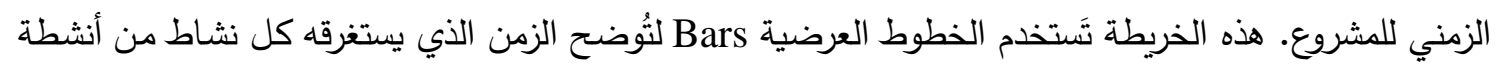

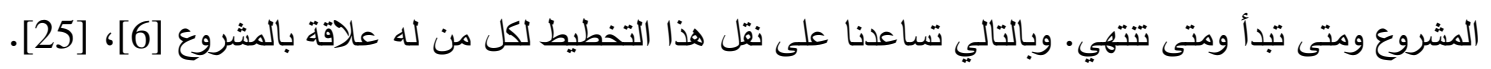

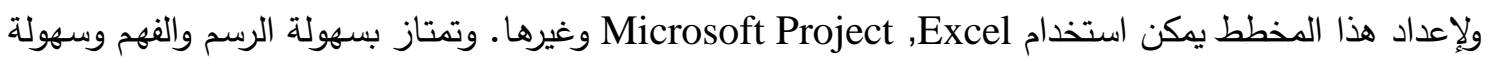
تعقب تقدم المشروع, أما عيوبها فإنها لا تحدد المسار الحرج بشكل واضـح وكذلك عدم ملائمتها للمشاريع الكبيرة بسبب وجود أنشطة تتكرر في عدة مسارات حرجة مما تسبب صعوبة تمثيلها بمخطط Gantt [23]. الثكل (3) يوضح مخطط Gantt لمشروع جسر الصرافية حسب اقتراح الباحثين.

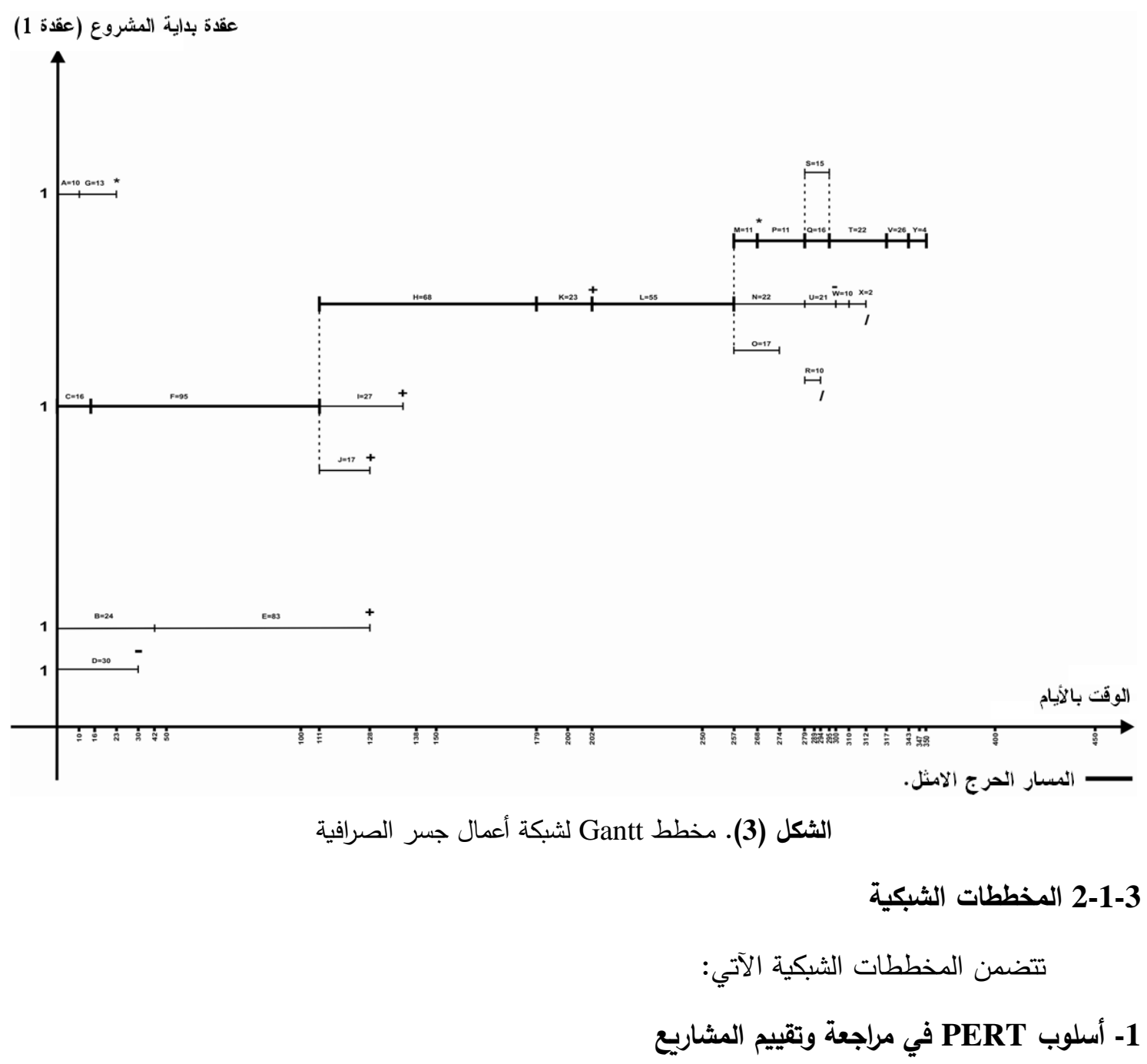

\section{Project Evaluation And Review Technique (PERT)}

هو أول نظام شبكي استخدم بثكل جماعي، تم تقديم هذا الأسلوب ما بين 1950-1958 من قبل شركة Hamilton. Booz-Allen بشركة Lockheed، بهدف تصميم طريقة يتم بها تخطيط ومتابعة صناعة وتطوير الصواريخ الباليستية بولاريس 
Polaris Missile

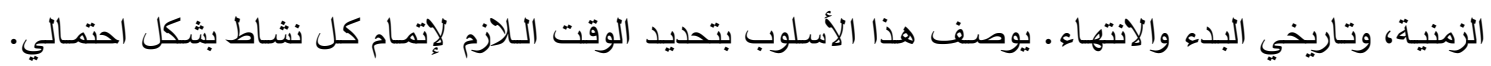

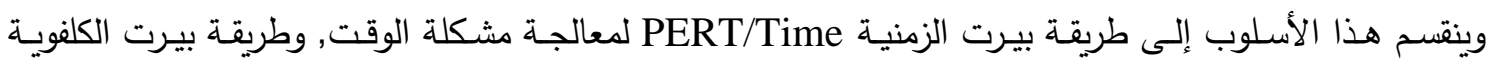
PERT/Cost

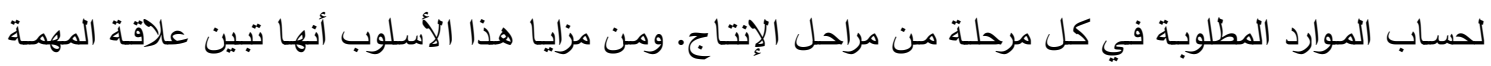
الاتكالية وسهولة تعقب المشروع وسهولة تحديد المسارات الحرجة. أما عيوبه فإنها لا تستخدم عمليا وذلك للاحتياج

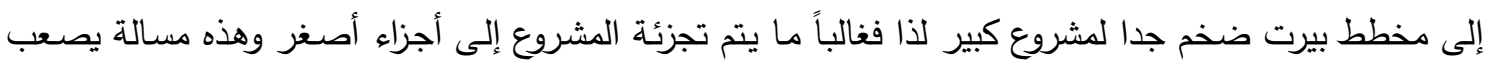

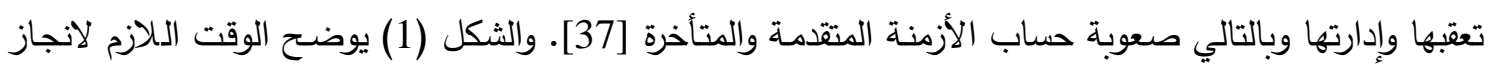
مشروع جسر الصرافية حسب أسلوب بيرت الزمني وهو 347 يوماً [7].

Critical Path Method (CPM) المسار الحرج

وهو أطول مسار خـلال الشبكة. أو أنه المسار الذي يستغرق أقصى وقت لانجاز المشروع من حدث البدايـة إلى حدث النهايـة. ظهر هذا الأسـلوب في عـام 1957 من قبـل المهندس emington-Rand والمهند M.R. Walker في شركة Dupont وذلك لغرض جدولة عمليات التعطل بسبب الصيانة في مصنع المواد الكيميائية. وبدأ استخدام الأسلوبين بيرت والمسار الحرج من قبل شركة فورد للسيارات

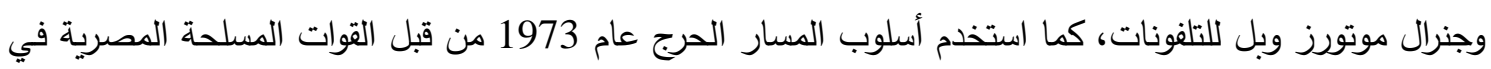
عبور قناة السويس واستخدم لأول مرة في العراق عام 1975 في بناء سد حمرين. إن الغرض الرئيسي من هذا

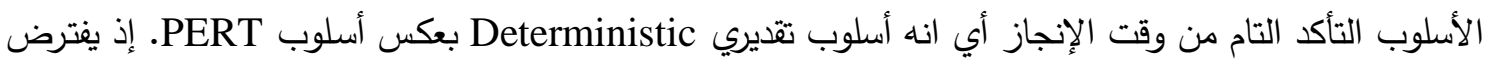
أسلوب المسار الحرج أن الوقت المتوقع لأداء العمليات المختلفة لإتمام المشروع معروف والعلاقية بين الموارد

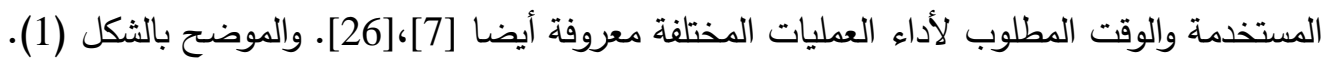

\section{Dynamic Programming البرمجة الديناميكية 3-1-3}

مصطلح الديناميكية تعني عدم الاستقرار على مر الزمن. إذ ارتبط ظهور أسلوب البرمجة الديناميكية في

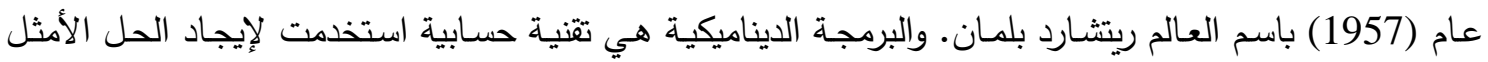

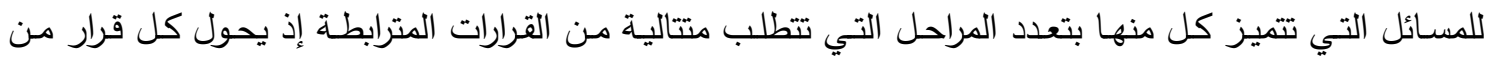
القرارات إلى عدة مسائل جزئية تمثل كل منها احد المراحل وبالمتغيرات التي تحويها ثم يتم التقدم بالحل من مرحلة

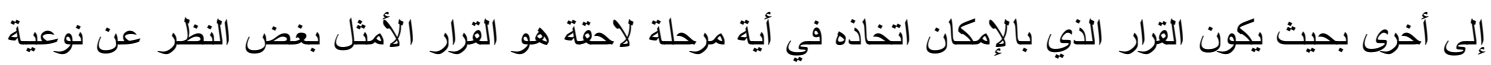

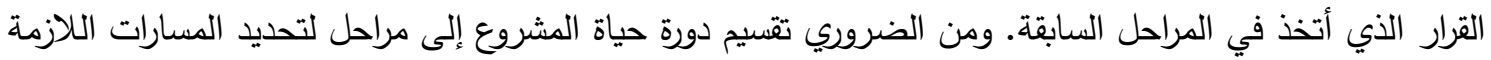

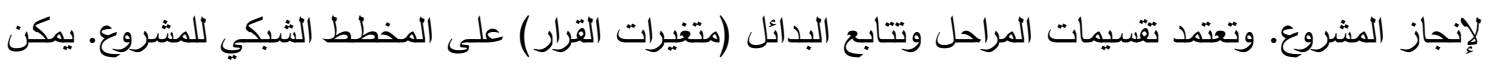
إيجاد الحد الأعلى الأمثل للوقت الخاص بانجداز المشروع بطريقتين[18], [30],_[20] :

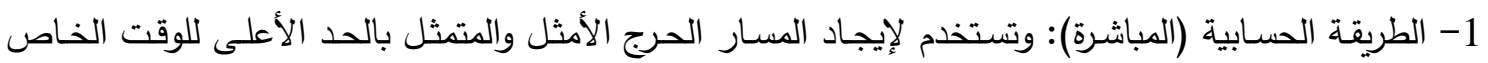
بانجاز المشروع. 
2- طريقة البرمجة الخطية: وتستخدم ضمن هذه الطريقة خوارزميتين هما الحل الأمامي والحل العكسي. وبما أن الهدف هو إيجاد الحد الأعلى الأمثل بالوقت الخاص بانجاز مشروع جسر الصرافية فقد تم إتباع الخوارزميتين

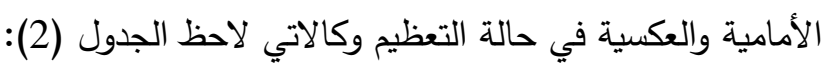
S = 1 ... المراحل، لمشروع جسر الصرافية S : S

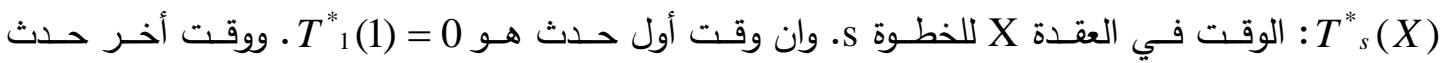

$$
\text { هو } \mathrm{T}_{13}{ }^{*}(20)=0
$$

X $\mathrm{T}(N, X)$

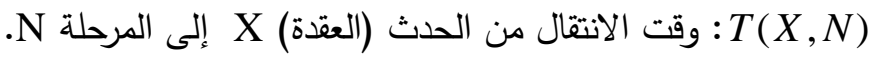

X متغير الحالة (State Variable) من مرحلة إلى أخرى. X

Forward Algorithm أ- الخوارزمية الأمامية

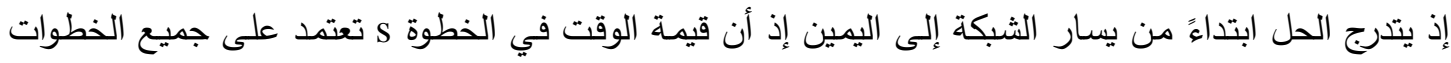
السابقة لها. إذ أن الحد الأعلى الأمثل للوقت الخاص بلتهان بانجاز مشروع جسر الصرافية يستغرق 274 يوما لاحظ

$T^{*}{ }_{s}(X)=\operatorname{Max}\left[T(N, X)+T^{*}{ }_{s-1}(N)\right]$ for $s=1,2, . ., 13$

$T^{*}{ }^{*}(1)=0$

$T^{*}{ }_{2}(2)=\operatorname{Max}\left[T(1,2)+T^{*}{ }_{1}(1)\right]=\operatorname{Max}[10+0]=10$

$T^{*}{ }_{2}(3)=\operatorname{Max}\left[T(1,3)+T^{*}{ }_{1}(1)\right]=\operatorname{Max}[16+0]=16$

$T^{*}{ }_{2}(4)=\operatorname{Max}\left[T(1,4)+T^{*}{ }_{1}(1)\right]=\operatorname{Max}[42+0]=42$

$T^{*}{ }_{2}(13)=\operatorname{Max}\left[T(1,13)+T^{*}{ }_{1}(1)\right]=\operatorname{Max}[30+0]=30$

$T^{*}{ }_{6}(9)=\operatorname{Max}\left[T(8,9)+T^{*}{ }_{5}(8)\right]=\operatorname{Max}[55+202]=257$

$T^{*}{ }_{7}(12)=\operatorname{Max}\left[T(9,12)+T^{*}{ }_{6}(9), T(11,12)+T^{*}{ }_{6}(11)\right]=\operatorname{Max}[17+257,0+0]=274$

$T^{*} 13(20)=52$

بackward Algorithm ب الخوارزمية العكسية

إذ يتدرج الحل من اليمين إلى اليسار إذ أن قيمة الوقت في الخطوة s تعتمد على جميع الخطوات اللاحقة

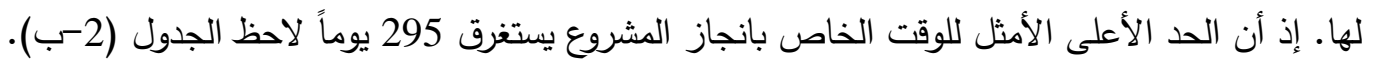
$T^{*}{ }_{s}(X)=\operatorname{Max}\left[T(X, N)+T_{s+1}^{*}(N)\right]$

$T^{*}{ }_{13}(20)=0$

$$
\begin{aligned}
T^{*}{ }_{1}(1) & =\operatorname{Max}\left[T(1,2)+T_{2}^{*}{ }_{2}(2), T(1,3)+T_{2}^{*}{ }_{2}(3), T(1,4)+T_{2}^{*}{ }_{2}(4), T(1,13)+T^{*}{ }_{2}(13)\right] \\
& =\operatorname{Max}[10+13,16+279,30+10]=\operatorname{Max}[23,295,125,40]=295
\end{aligned}
$$


الجدول (2). الحل اليدوي لطريقة البرمجة الديناميكية

الجدول (2-أ): الخوارزمية الأمامية

\begin{tabular}{|c|c|c|c|c|c|c|c|c|c|c|c|c|c|c|c|c|c|c|c|c|}
\hline (X) & 1 & 2 & 3 & 4 & 5 & 6 & 7 & 8 & 9 & 10 & 11 & 12 & 13 & 14 & 15 & 16 & 17 & 18 & 19 & 20 \\
\hline 1 & 0 & $*$ & $*$ & $*$ & $*$ & $*$ & $*$ & $*$ & $*$ & * & $*$ & $*$ & * & $*$ & $*$ & $*$ & $*$ & $*$ & $*$ & $*$ \\
\hline 2 & $*$ & 10 & 16 & 42 & $*$ & $*$ & $*$ & $*$ & $*$ & $*$ & $*$ & $*$ & 30 & $*$ & $\%$ & $*$ & $*$ & $*$ & $\%$ & $\%$ \\
\hline 3 & $*$ & $*$ & $*$ & $*$ & 111 & $*$ & $*$ & $*$ & $*$ & $*$ & $*$ & $*$ & $*$ & $*$ & $*$ & $*$ & $*$ & $*$ & $*$ & $*$ \\
\hline 4 & $*$ & $*$ & $*$ & $*$ & 179 & 128 & $*$ & $*$ & $*$ & $*$ & $*$ & $*$ & $*$ & $*$ & $*$ & $*$ & $*$ & $*$ & $*$ & $*$ \\
\hline 5 & $*$ & $*$ & $*$ & $*$ & $*$ & $*$ & $*$ & 202 & $*$ & $*$ & $*$ & $*$ & $*$ & $*$ & $*$ & $*$ & $*$ & $*$ & $*$ & $\%$ \\
\hline 6 & $*$ & $*$ & $*$ & $*$ & $*$ & $*$ & $*$ & $*$ & 257 & $*$ & $*$ & $*$ & $*$ & $*$ & $*$ & $*$ & $*$ & $*$ & $*$ & $*$ \\
\hline 7 & $*$ & $*$ & $*$ & $*$ & $*$ & $*$ & $*$ & $*$ & $*$ & 268 & $*$ & 274 & $*$ & $*$ & $*$ & $*$ & $*$ & $*$ & $*$ & $\%$ \\
\hline 8 & $*$ & $*$ & $*$ & $*$ & $*$ & $*$ & $*$ & $*$ & $*$ & $*$ & 22 & $*$ & $*$ & $*$ & $*$ & $*$ & $*$ & $*$ & $*$ & $\%$ \\
\hline 9 & $*$ & $*$ & $*$ & $*$ & $*$ & $*$ & $\%$ & $*$ & $*$ & $*$ & $*$ & $*$ & $*$ & 32 & 11 & $*$ & $*$ & $*$ & $*$ & $*$ \\
\hline 10 & $*$ & * & $*$ & $*$ & $*$ & $*$ & $*$ & $*$ & $*$ & $*$ & $*$ & $*$ & $*$ & $*$ & $*$ & 27 & 27 & $*$ & $*$ & $*$ \\
\hline 11 & $*$ & $*$ & $*$ & $*$ & $*$ & $*$ & $*$ & $*$ & $*$ & $*$ & $*$ & $*$ & $*$ & $*$ & $*$ & $*$ & $*$ & 22 & $*$ & $*$ \\
\hline 12 & * & $*$ & $*$ & $*$ & $*$ & $*$ & $*$ & $*$ & * & $*$ & $*$ & $*$ & $*$ & $*$ & $*$ & $*$ & $*$ & $*$ & 48 & $*$ \\
\hline 13 & $*$ & $*$ & $*$ & $*$ & $*$ & $*$ & $*$ & $*$ & $*$ & $*$ & $*$ & $*$ & $*$ & * & $*$ & $*$ & $*$ & $*$ & $*$ & 52 \\
\hline
\end{tabular}

الجدول(2-ب). الخوارزمية العكسية

\begin{tabular}{|c|c|c|c|c|c|c|c|c|c|c|c|c|c|c|c|c|c|c|c|c|}
\hline$(\mathbf{X})$ & 1 & 2 & 3 & 4 & 5 & 6 & 7 & 8 & 9 & 10 & 11 & 12 & 13 & 14 & 15 & 16 & 17 & 18 & 19 & 20 \\
\hline 1 & 295 & $*$ & $*$ & $*$ & $*$ & * & $*$ & * & $*$ & * & * & * & * & $*$ & * & * & * & * & * & $*$ \\
\hline 2 & $*$ & 13 & 279 & 83 & $*$ & $*$ & * & $*$ & $*$ & $*$ & $*$ & $*$ & 10 & * & $*$ & $*$ & $*$ & $*$ & $*$ & $*$ \\
\hline 3 & $*$ & $*$ & $*$ & $*$ & 184 & $*$ & $*$ & $*$ & $*$ & $*$ & $*$ & $*$ & $*$ & $*$ & $*$ & $*$ & $\%$ & $*$ & $*$ & $*$ \\
\hline 4 & $*$ & $*$ & $*$ & $*$ & $*$ & 116 & 93 & $*$ & $*$ & $*$ & $*$ & $*$ & $*$ & $*$ & $*$ & $*$ & $*$ & $*$ & $*$ & $*$ \\
\hline 5 & $*$ & $*$ & $*$ & $*$ & $*$ & $*$ & $*$ & 93 & $*$ & $*$ & $*$ & $*$ & $*$ & $*$ & $*$ & $*$ & $\%$ & $*$ & $*$ & $*$ \\
\hline 6 & $*$ & $*$ & $*$ & $*$ & $*$ & $*$ & $*$ & $*$ & 38 & $*$ & $*$ & $*$ & $*$ & $*$ & $*$ & $*$ & $*$ & $*$ & $*$ & $*$ \\
\hline 7 & $*$ & $*$ & $*$ & $*$ & $*$ & $*$ & $*$ & $*$ & $*$ & 11 & $*$ & 21 & $*$ & $*$ & $*$ & $*$ & $*$ & $*$ & $*$ & $*$ \\
\hline 8 & $*$ & $*$ & $*$ & $*$ & $*$ & $*$ & $*$ & $*$ & $*$ & $*$ & 12 & $*$ & * & $*$ & $*$ & * & $*$ & $*$ & $*$ & $*$ \\
\hline 9 & $*$ & $*$ & $*$ & $*$ & $*$ & $*$ & $*$ & $*$ & $*$ & $*$ & $*$ & $*$ & $*$ & 2 & 67 & $*$ & $*$ & $*$ & $*$ & $\%$ \\
\hline 10 & $*$ & $*$ & $*$ & $*$ & $*$ & $*$ & $*$ & $*$ & $*$ & $*$ & $*$ & $*$ & $*$ & $*$ & $*$ & $\mathbf{0}$ & 52 & $*$ & $*$ & $*$ \\
\hline 11 & $*$ & $*$ & $*$ & $*$ & $*$ & $*$ & $*$ & $*$ & $*$ & $*$ & $*$ & $*$ & $*$ & $*$ & $*$ & $*$ & $*$ & 30 & * & $*$ \\
\hline 12 & $*$ & $*$ & $*$ & $*$ & $*$ & $\%$ & $*$ & $*$ & $*$ & $*$ & $*$ & $*$ & $*$ & $*$ & $*$ & $*$ & $*$ & $*$ & 4 & $*$ \\
\hline 13 & $*$ & $*$ & $*$ & $*$ & $*$ & $*$ & $*$ & $*$ & $*$ & $*$ & $*$ & $*$ & $*$ & $*$ & $*$ & $*$ & $*$ & $*$ & $*$ & 2 \\
\hline
\end{tabular}

*: not connected

4-1-3 تطبيق البرنامج الجاهز WinQSB:

طبق البرنـامج على مشروع جسر الصـرافية, إذ حولت الثبكة يدوياً من قبل الباحثين إلى دالة هدف

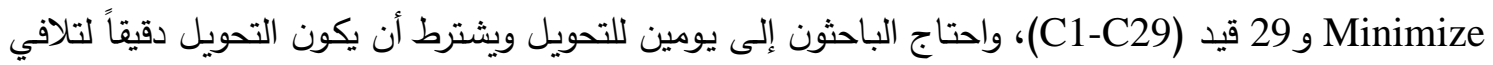

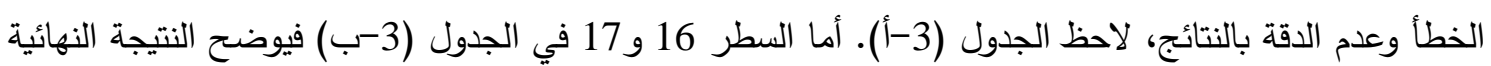

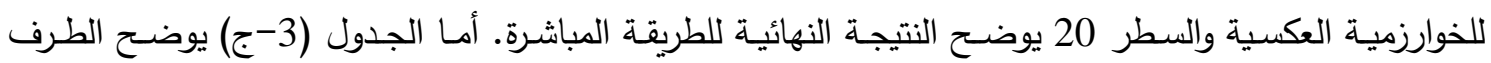
الأيسر للقيود والمثثل بالعمود left Hand Side والعمود Right Hand Side يمثل الموارد المتاحة أمسا All.max RHS All.min RHS المسألة التي تم حلها إضافة إلى عدد المحاولات والوقت المستغرق للحل. All 
الجدول (3). تطبيق برنامج WinQSB لحل مسألة شبكة أعمال جسر الصرافية

الجدول (3-1). الواجهة (1) إدخال دالة الهدف والقيود لمسألة شبكة أعمال جسر الصرافية يدوياً

\begin{tabular}{|c|c|}
\hline & OBJ/Constraint/Bound \\
\hline Minimize & $x 20-x 1$ \\
\hline C1 & $x 2-x 1>=10$ \\
\hline C2 & $\times 3-x 1>=16$ \\
\hline C3 & $x 3-x 2>=0$ \\
\hline C4 & $x 4-x 1>=42$ \\
\hline C5 & x13-x1>=30 \\
\hline C6 & $x 5-x 3>=95$ \\
\hline C7 & $x 6-x 5>=68$ \\
\hline C8 & $x 7-x 5>=17$ \\
\hline C9 & $x 8-x 5>=27$ \\
\hline C10 & $x 8-x 6>=23$ \\
\hline C11 & $x 8-x 7>=0$ \\
\hline C12 & $x 7-x 4>=83$ \\
\hline C13 & $x 9-x 8>=55$ \\
\hline C14 & $x 10-x 2>=13$ \\
\hline C15 & $x 10-x 9>=11$ \\
\hline C16 & x11-x9>=22 \\
\hline C17 & $x 12-x 9>=17$ \\
\hline C18 & $\times 12-x 11>=0$ \\
\hline C19 & $x 15-x 10>=11$ \\
\hline C20 & $\times 13-x 12>=21$ \\
\hline C21 & $\times 14-x 13>=10$ \\
\hline C22 & $\times 14-x 11>=10$ \\
\hline C23 & $x 17-x 15>=15$ \\
\hline C24 & $x 16-x 15>=16$ \\
\hline C25 & $\times 17-x 16>=0$ \\
\hline C26 & $x 18-x 17>=22$ \\
\hline C27 & $x 19-x 18>=26$ \\
\hline C28 & $x 20-x 19>=4$ \\
\hline C29 & $\times 20-x 14>=2$ \\
\hline Integer: & \\
\hline Binary: & \\
\hline
\end{tabular}

الجدول (3-ب). الواجهة (2) النتيجة النهائية للطريقة المباشرة وللخوارزمية العكسية

\begin{tabular}{|c|c|c|c|c|c|c|c|}
\hline & $\begin{array}{c}\text { Solution } \\
\text { Value }\end{array}$ & $\begin{array}{l}\text { Unit Cost or } \\
\text { Profit c[i] }\end{array}$ & $\begin{array}{c}\text { Total } \\
\text { Contribution }\end{array}$ & $\begin{array}{l}\text { Reduced } \\
\text { Cost }\end{array}$ & $\begin{array}{l}\text { Basis } \\
\text { Status }\end{array}$ & $\begin{array}{l}\text { Allowable } \\
\text { Min. c[i] }\end{array}$ & $\begin{array}{l}\text { Allowable } \\
\text { Max. c[i] }\end{array}$ \\
\hline 1 & $\mathbf{0}$ & -1.0000 & 0 & $\mathbf{0}$ & at bound & -1.0000 & M \\
\hline 2 & 16.0000 & 0 & 0 & $\mathbf{0}$ & basic & 0 & $\mathbf{0}$ \\
\hline 3 & 16.0000 & 0 & $\mathbf{0}$ & $\mathbf{0}$ & basic & 0 & M \\
\hline 4 & 119.0000 & $\mathbf{0}$ & 0 & 0 & basic & 0 & $\mathbf{0}$ \\
\hline 5 & 111.0000 & $\mathbf{0}$ & 0 & $\mathbf{0}$ & basic & 0 & M \\
\hline 6 & 179.0000 & $\mathbf{0}$ & $\mathbf{0}$ & 0 & basic & $\mathbf{0}$ & $\mathbf{M}$ \\
\hline 7 & 202.0000 & 0 & 0 & 0 & basic & 0 & $\mathbf{0}$ \\
\hline 8 & 202.0000 & 0 & 0 & 0 & basic & 0 & $\mathbf{M}$ \\
\hline 9 & 257.0000 & $\mathbf{0}$ & 0 & 0 & basic & $\mathbf{0}$ & $\mathbf{M}$ \\
\hline 10 & 268.0000 & 0 & 0 & 0 & basic & 0 & M \\
\hline 11 & 314.0000 & 0 & 0 & 0 & basic & 0 & $\mathbf{0}$ \\
\hline 12 & 314.0000 & $\mathbf{0}$ & $\mathbf{0}$ & 0 & basic & 0 & $\mathbf{0}$ \\
\hline 13 & 335.0000 & 0 & 0 & $\mathbf{0}$ & basic & 0 & 0 \\
\hline 14 & 345.0000 & 0 & 0 & 0 & basic & 0 & 0 \\
\hline 15 & 279.0000 & $\mathbf{0}$ & $\mathbf{0}$ & 0 & basic & $\mathbf{0}$ & M \\
\hline 16 & 295.0000 & 0 & 0 & $\mathbf{0}$ & basic & 0 & M \\
\hline 17 & 295.0000 & 0 & 0 & $\mathbf{0}$ & basic & 0 & M \\
\hline 18 & 317.0000 & 0 & $\mathbf{0}$ & 0 & basic & $\mathbf{0}$ & M \\
\hline 19 & 343.0000 & $\mathbf{0}$ & $\mathbf{0}$ & $\mathbf{0}$ & basic & $\mathbf{0}$ & M \\
\hline \multirow[t]{2}{*}{20} & 347.0000 & 1.0000 & 347.0000 & $\mathbf{0}$ & basic & 1.0000 & M \\
\hline & Function & [Min.] = & 347.0000 & (Note: & Alternate & Solution & Exists!!] \\
\hline
\end{tabular}




$$
\text { الجدول (3-ج). الواجهة (3) الحد الأدنى والأعلى للقيود المتاحة }
$$

\begin{tabular}{|c|c|c|c|c|c|c|c|}
\hline & $\begin{array}{l}\text { Left Hand } \\
\text { Side }\end{array}$ & Direction & $\begin{array}{c}\text { Right Hand } \\
\text { Side }\end{array}$ & $\begin{array}{c}\text { Slack } \\
\text { or Surplus }\end{array}$ & $\begin{array}{c}\text { Shadow } \\
\text { Price }\end{array}$ & $\begin{array}{l}\text { Allowable } \\
\text { Min. RHS }\end{array}$ & $\begin{array}{l}\text { Allowable } \\
\text { Max. RHS }\end{array}$ \\
\hline 1 & 16.0000 & $>=$ & 10.0000 & 6.0000 & o & $-M$ & 16.0000 \\
\hline 2 & 16.0000 & $>=$ & 16.0000 & o & 1.0000 & 10.0000 & M \\
\hline 3 & o & $>=$ & 0 & $\mathbf{0}$ & o & -239.0000 & 6.0000 \\
\hline 4 & 119.0000 & $>=$ & 42.0000 & 77.0000 & o & $-M$ & 119.0000 \\
\hline 5 & 335.0000 & $>=$ & 30.0000 & 305.0000 & $\mathbf{0}$ & $-M$ & 335.0000 \\
\hline 6 & 95.0000 & $>=$ & 95.0000 & o & 1.0000 & 18.0000 & M \\
\hline 7 & 68.0000 & $>=$ & 68.0000 & o & 1.0000 & 4.0000 & M \\
\hline $\mathbf{8}$ & 91.0000 & $>=$ & 17.0000 & 74.0000 & o & $-M$ & 91.0000 \\
\hline $\mathbf{9}$ & 91.0000 & $>=$ & 27.0000 & 64.0000 & $\mathbf{0}$ & $-\mathbf{M}$ & 91.0000 \\
\hline 10 & 23.0000 & $>=$ & 23.0000 & o & 1.0000 & -41.0000 & M \\
\hline 11 & o & $>=$ & 0 & $\mathbf{0}$ & o & $-\mathbf{M}$ & 74.0000 \\
\hline 12 & 83.0000 & $>=$ & 83.0000 & 0 & $\mathbf{0}$ & $-\mathbf{M}$ & 160.0000 \\
\hline 13 & 55.0000 & $>=$ & 55.0000 & $\mathbf{0}$ & 1.0000 & -184.0000 & M \\
\hline 14 & 252.0000 & $>=$ & 13.0000 & 239.0000 & o & $-M$ & 252.0000 \\
\hline 15 & 11.0000 & $>=$ & 11.0000 & o & 1.0000 & -24.0000 & M \\
\hline 16 & 57.0000 & $>=$ & 22.0000 & 35.0000 & D & $-\mathbf{M}$ & 57.0000 \\
\hline 17 & 57.0000 & $>=$ & 17.0000 & 40.0000 & o & $-M$ & 57.0000 \\
\hline 18 & o & $>=$ & 0 & o & o & -21.0000 & 35.0000 \\
\hline 19 & 11.0000 & $>=$ & 11.0000 & $\mathbf{0}$ & 1.0000 & -24.0000 & M \\
\hline 20 & 21.0000 & $>=$ & 21.0000 & $\mathbf{0}$ & o & o & 56.0000 \\
\hline 21 & 10.0000 & $>=$ & 10.0000 & o & 0 & -11.0000 & 45.0000 \\
\hline 22 & 31.0000 & $>=$ & 10.0000 & 21.0000 & 0 & $-M$ & 31.0000 \\
\hline 23 & 16.0000 & $>=$ & 15.0000 & 1.0000 & $\mathbf{0}$ & $-M$ & 16.0000 \\
\hline 24 & 16.0000 & $>=$ & 16.0000 & o & 1.0000 & 15.0000 & M \\
\hline 25 & o & $>=$ & o & o & 1.0000 & -1.0000 & M \\
\hline 26 & 22.0000 & $>=$ & 22.0000 & o & 1.0000 & -13.0000 & M \\
\hline 27 & 26.0000 & $>=$ & 26.0000 & o & 1.0000 & -9.0000 & $\mathbf{M}$ \\
\hline 28 & 4.0000 & $>=$ & 4.0000 & $\mathbf{0}$ & 1.0000 & -31.0000 & $M$ \\
\hline 29 & 2.0000 & $>=$ & 2.0000 & o & o & $-M$ & 37.0000 \\
\hline
\end{tabular}

الجدول (3-د). الواجهة (4) تقرير عن نوع المسألة وعدد المحاولات والوقت المستغرق

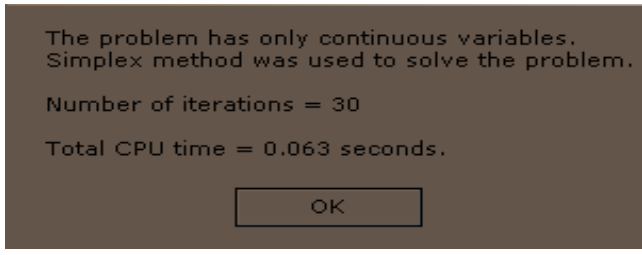

2-3 أسباب اختيار الخوارزمية الجينية وملائمتها لحل مسألة جدولة المشاريع

هناك عدة تقنيات ذكائية لحل مسألة الجدولة [9]،[35]. ومنها الخوارزمية الجينية التي ابتكرت من قبل

العالم John Holland عام 1975. والسببين الرئيسيين لاختيار الخوارزمية الجينية:

$$
\text { 1- تمتاز الخوارزمية الجينية بالاتي [29]، [31]: }
$$

أ- الرصانة Robustness: يجب أن تكون معتمدة reliable وقادرة على الاقتراب من الحل ابتداءً من أي

نقطة معطاة (كأن يكون الحل الأمثل يعد نقطة بداية لتحسين الحل).

ب- العمومية Generality: لا يتفرض الحد من قيود النموذج ودالة الهدف.

ج- الدقة Accuracy: المهارة والقدرة على الاقتراب من حل امثل ودقيق وهذا لا يتطلب خبرة في الأمثلية

$$
\text { وبحوث العمليات. }
$$

د- سهولة التطبيق والاستخدام: سواء من قبل الخبير أو الأقل خبرة.

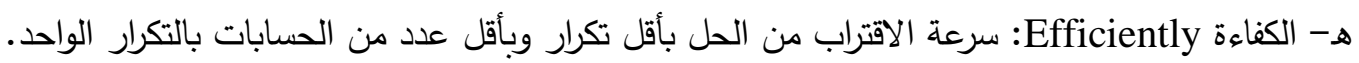

2- تكون الخوارزمية الجينية فعالة لحل المسائل التي تحقق واحدة أو أكثر من الحالات التي على أساسها يتت

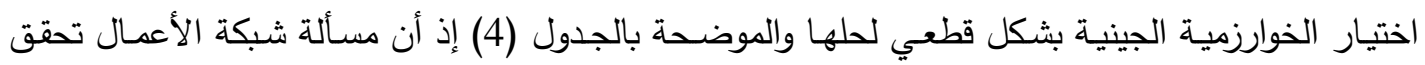

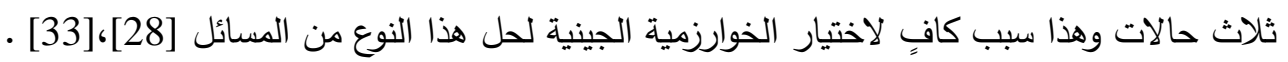


الجدول (4). ملائمة حل مسألة شبكة الأعمال باستخدام الخوارزمية الجينية

\begin{tabular}{|c|c|c|c|c|c|}
\hline 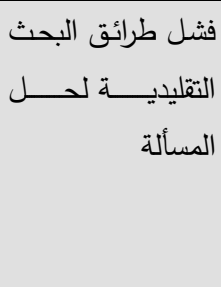 & 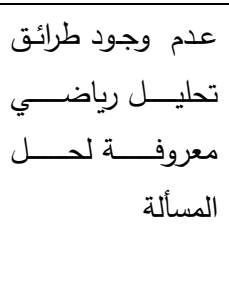 & 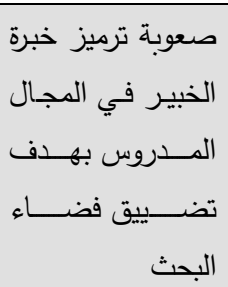 & 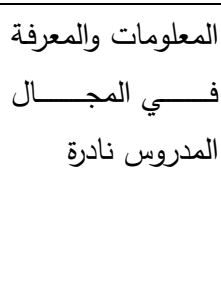 & فضاء البحث هائل & الخوارزمية الجينية \\
\hline$\angle$ & & $\angle$ & & $\angle$ & شبكة الأعمال \\
\hline
\end{tabular}

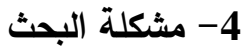

من المعوقات التي واجهت الباحثين في تصميم الخوارزمية الجينية لحل مسائل الأمثلية المتعلقة بشبكات الأعمال. وعلى اعتبار أن كل كروموسوم يمثل مساراً حرجاً واحداً فستؤدي عملية التزاوج أو الطفرة إلى تحطيه

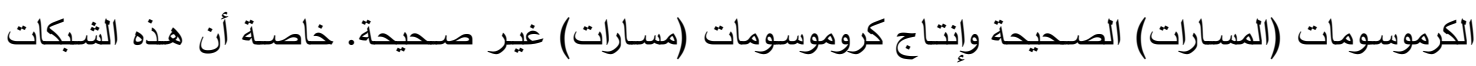
تختلف من ناحية عدد المراحل وعدد العقد في كل مرحلة. تكمن المشاكل بما يلي [5]، [29]:

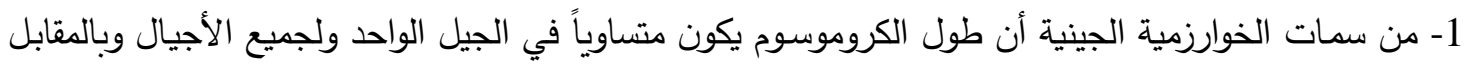
فالمسارات الحرجة تكون مختلفة الأطوال (كل مسار يحوي على عدد مختلف من العقد) وبالتالي صعوبة تمثيل المسارات الحرجة المختلفة الأطوال ككروموسومات متساوية الأطوال (لاحظ المسارات في الثبكة).

$1-13-14-20$

$1-2-10-9-11-14-20$

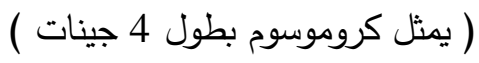

( يمثل كروموسوم بطول 7 جينات )

وهكذا نلاحظ أن هنالك عدة مسارات ذات أطوال مختلفة في شبكة الأعمال. 2- الخوارزمية الجينية تعتمد على توليد سلسلة عشوائية من العقد كجيل أولي وأن عملية أنثاء هذا الجيل بشكل فئل فئل عشوائي ستولد كروموسومات لمسارات لا تطابق أي مسار من مسارات الشبكة.

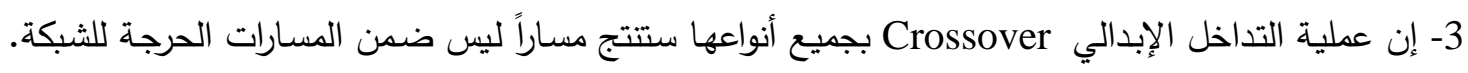

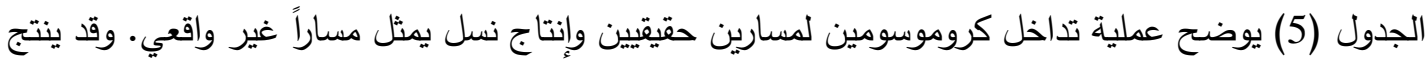
نسل تتكرر قيم جيناته فيمثل مساراً يحوي على عقدة (أو عقد) تتكرر لأكثر من مرة وهذا يخالف احد شروط تمثيل المخطط الثبكي للمشروع: الجدول (5). عملية التداخل الإبدالي باستخدام نقطتين

\begin{tabular}{|c|c|c|c|c|c|c|c|c|c|}
\hline 1 & 3 & 5 & 6 & 8 & 9 & 11 & 14 & 20 & الكروموسوم الأول \\
\hline 1 & 3 & 5 & 8 & 9 & 12 & 13 & 14 & 20 & الكروموسوم الثاني \\
\hline 1 & 3 & 5 & 8 & 9 & 12 & 11 & 14 & 20 & النسل الناتج \\
\hline
\end{tabular}

4- إن عملية أجراء الطفرة بجميع أنواعها ستتنج كروموسوماً غير مطابق لأي مسار من مسارات شبكة المشروع,

لاحظ الجدول (6).

الجدول (6). عملية الطفرة بتبديل جينين

\begin{tabular}{|l|l|l|l|l|l|l|l|l|l|}
\hline 1 & 3 & 5 & 8 & 9 & 12 & 11 & 14 & 20 & النسل قبل الطفرة \\
\hline 1 & 3 & 5 & 14 & 9 & 12 & 11 & 8 & 20 & الطعرة \\
\hline
\end{tabular}


1- استخدام التقنيات الذكائية لصنع القرار عوضا عن التقنيات التقليدية لحل مسائل بحوث العمليات وخاصـة فيما

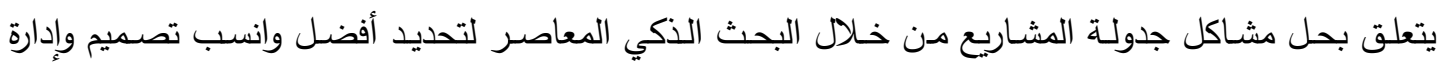
وتشغيل النظام تحت شروط تتطلب توزيع الموارد النادرة.

2- اقتراح وتصـيم خوارزميـة ذكيـة كفوءة لجدولـة المشـاريع GAOCPN كتطوير للخوارزميـة الجينيـة التقليديـة لتخطي مشكلة المسارات المختلفة مع ثبوت طول الكروموسوم وتجاوز مشكلة توليد سلسلة من الأنشطة الغير واقعية وبالتالي تجاوز جميع المشاكل المبينة في مشكلة البحث. 3- تطبيق GAOCPN على كافة أنواع شبكات الأعمال مهما يكن عدد مراحلها وعدد العقد في كل مرحلة ومهما يكن عدد وأطوال مساراتها. 4- قياس جودة GAOCPN بتحقيق وتطبيق مقاييس الجودة النوعية للخوارزمية الجينية, الرصانة Robustness,

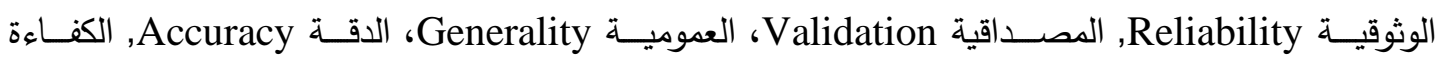

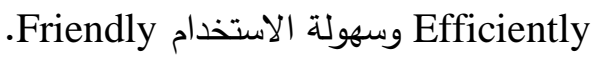
5- تقديم أنمـوذج تخمينـي ذكي ومعاصـر للبرمجـة الديناميكيـة والممثل بالصـندوق الأسـود GAOCPN.EXE

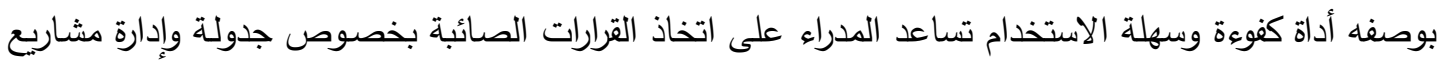
الأعمال وانجازها بطرائق تعتمد على تكنولوجيا العصر والتقنيات الذكائية. 6- تطوير مجال بحوث العمليات باعتماده على التقنيات الذكائية بدلا من التقليدية.

\section{6- 6رضية البحث}

بما أن شبكة الأعمال تحوي n من العقد وكل عقدة لها عدة تفرعات فقد تم اعتماد عدة فرضيات لإنجاز

$$
\text { البحث منها: }
$$

1- بعض البحوث استخدم المصفوفة لإنشاء الثبكة ومعاييرها (كلفة، وقت، ربح) وكالاتي [32]:

$$
\begin{aligned}
& \operatorname{net}(\mathrm{i}, \mathrm{j})=0
\end{aligned}
$$

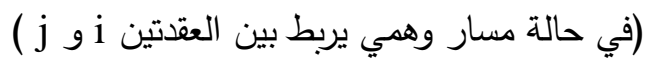

$$
\begin{aligned}
& \operatorname{net}(\mathrm{i}, \mathrm{j})>0
\end{aligned}
$$

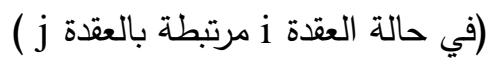

$$
\begin{aligned}
& \operatorname{net}(i, j)=-1
\end{aligned}
$$

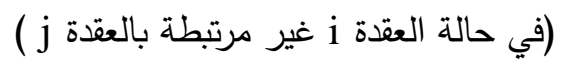

إن هيكل البيانات net netn تحتاج إلى مساحة خزنية في الذاكرة تتاسب طرديا مع حجم شبكة الأعمال، أما في

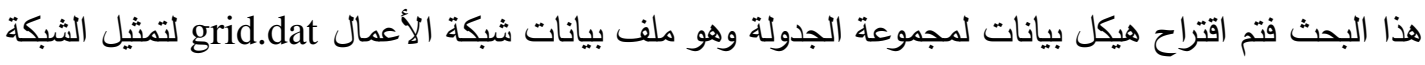
بأقل مساحة خزنية ممكنة، وذلك باعتماد الحالة الأولى والثانية فقط لاحظ الجدول (7). وتمتاز فرضية البحث بما يخص هيكل البيانات المقترح للشبكة مقارنة مع غيرها لتمثيل شبكة الأعمال بالحاسوب بأقل سعة خزنية.

\begin{tabular}{|c|c|c|}
\hline \multirow{2}{*}{$\begin{array}{c}\text { العقدة } \\
\text { i }\end{array}$} & \multirow[t]{2}{*}{ عدد العقد المرتبطة بها } & العقدة التالية للعقدة i \\
\hline & & قيمة النشاط \\
\hline 1 & 4 & $\begin{array}{llll}2 & 3 & 4 & 13\end{array}$ \\
\hline & & $\begin{array}{llll}10 & 16 & 42 & 30\end{array}$ \\
\hline 2 & 2 & $\begin{array}{ll}3 & 10\end{array}$ \\
\hline & & $\begin{array}{ll}0 & 13 \\
\end{array}$ \\
\hline 3 & 1 & 5 \\
\hline & & 95 \\
\hline 4 & 1 & 7 \\
\hline & & 83 \\
\hline 5 & 3 & 6 \\
\hline
\end{tabular}
الجدول (7). هيكل بيانات الملف grid.dat لمجموعة جدولة شبكة أعمال جسر الصرافية 


\begin{tabular}{|c|c|c|}
\hline & & $\begin{array}{lll}68 & 17 & 27 \\
\end{array}$ \\
\hline \multirow[t]{2}{*}{6} & 1 & 8 \\
\hline & & 23 \\
\hline \multirow[t]{2}{*}{7} & 1 & 8 \\
\hline & & 0 \\
\hline \multirow[t]{2}{*}{8} & 1 & 9 \\
\hline & & 55 \\
\hline \multirow[t]{2}{*}{9} & 3 & $\begin{array}{llll}10 & 11 & 12 \\
\end{array}$ \\
\hline & & $\begin{array}{lll}11 & 22 & 17\end{array}$ \\
\hline \multirow[t]{2}{*}{10} & 1 & 15 \\
\hline & & 11 \\
\hline \multirow[t]{2}{*}{11} & 2 & 1214 \\
\hline & & $\begin{array}{ll}0 & 10 \\
\end{array}$ \\
\hline \multirow[t]{2}{*}{12} & 1 & 13 \\
\hline & & 21 \\
\hline \multirow[t]{2}{*}{13} & 1 & 14 \\
\hline & & 10 \\
\hline \multirow[t]{2}{*}{14} & 1 & 20 \\
\hline & & 2 \\
\hline \multirow[t]{2}{*}{15} & 2 & $\begin{array}{ll}16 & 17 \\
\end{array}$ \\
\hline & & $16 \quad 15$ \\
\hline \multirow[t]{2}{*}{16} & 1 & 17 \\
\hline & & 0 \\
\hline \multirow[t]{2}{*}{17} & 1 & 18 \\
\hline & & 22 \\
\hline \multirow[t]{2}{*}{18} & 1 & 19 \\
\hline & & 26 \\
\hline \multirow[t]{3}{*}{19} & 1 & 20 \\
\hline & & 4 \\
\hline & 0 & \\
\hline
\end{tabular}

2- بما أن مسارات الثبكة مختلفة الأطوال وللحصول على كروموسومات تمثل مسارات الشبكة بشكل صحيح فتم

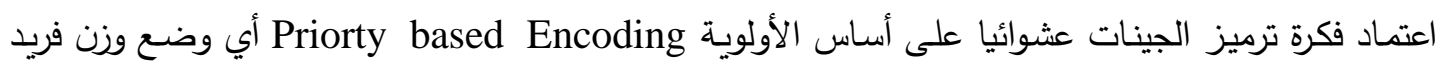
(وحيد) لكل جين في الكروموسوم الواحد وتتغير هذه الأوزان من كروموسوم لآخر ومن جيل لآخر الآنس [22].

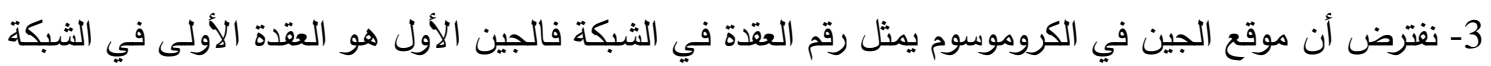

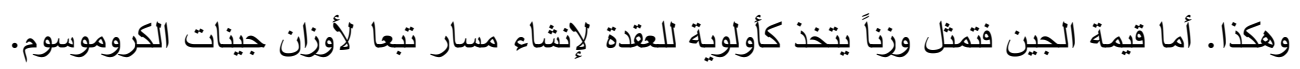

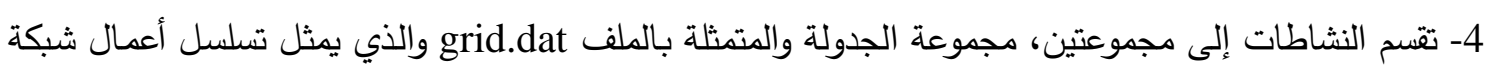
المشروع. ومجموعة القرار وهي الكروموسومات التي تمثل قائمة الأولويات لكل النشاطات المطلوب جدولتها,

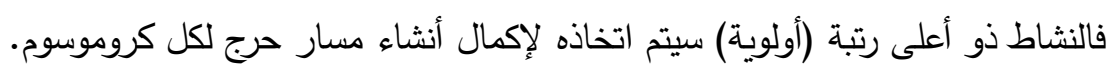

GAOCPN Tميزات وخصائص تمتاز خوارزمية GAOCPN بكونها كفوءة ودقيقة مقارنة مع الحلول التقليدية لبحوث العمليات (البرمجة الديناميكية) ومع الخوارزمية الجينية للبائع المتجول GATSP ولعدة أسباب موضحة كونة أدناه:

\section{1-7 مقارنة GAOCPN مع البرمجة الايناميكية}

GAOCPN ليست مقيدة بنـوع معين مـن شبكات الأعمـال كمـا هو الحسال في طريقـة البرمجـة الخطيـة

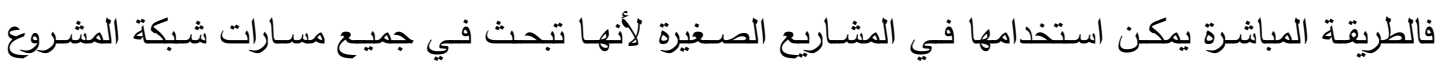
ولا ينجح تطبيقها في المشاريع الضخمة لأنها تستغرق وقتاً وجهداً كبيراً ولهذا يلجأ خبير (Search Space) بحوث العمليات إلى استخدام إحدى طرائق البرمجة الخطية لأنها لا تبحث في جميع المسارات وإنما تبحث في

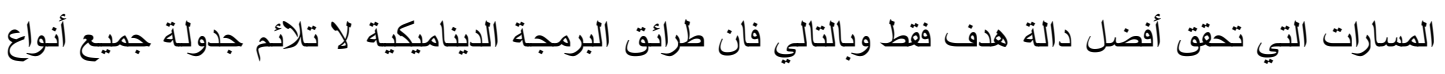


الشبكات، غير أن GAOCPN يمكن تطبيقها على جميع أنواع الثبكات ومنها الشبكات التي لا تقدر على

حلها البرمجة الخطية.

2- إن تطبيق GAOCPN لا تتطلب استشـارة خبير بحوث العمليات كما هو الحال في التقنيات التقليدية التي

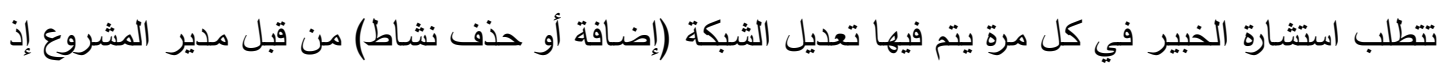
يتطلب ذلك جهداً حسابياً ووقتاً إضافياً للاتصال بالخبير المختص لإعادة تصميم الأنموذج وإيجاد امثل مسار حرج بعد تعديل الثبكة [16]. لاحظ الثكل (1) والجدولين (1 و3-3أ).

لا تلطلب تمثيل شبكة المشروع بوصفها أنموذج برمجة خطية (دالة هدف وقيود) لحله بينما

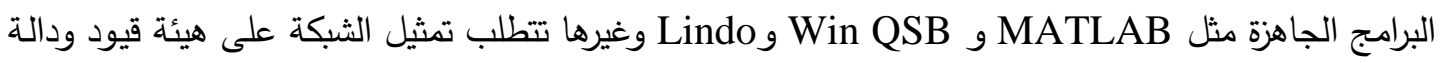
هدف لحلها وعملية التحويل هذه تحتاج إلى خبير يستوعب الثبكة بكفاءة ليمثلها بثكل دقيق وصحيح بوصفها

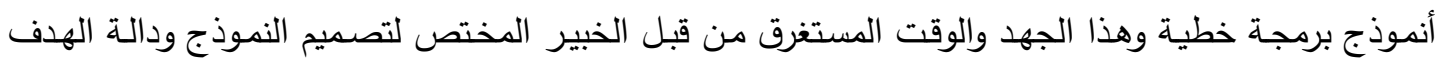
وجميع قيود النموذج، كل هذا يزداد طرديا مع درجة تعقيد الثبكة وكذلك يزداد طردياً مع المشاريع ذات التوسع

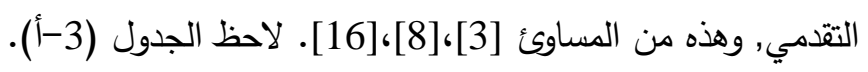

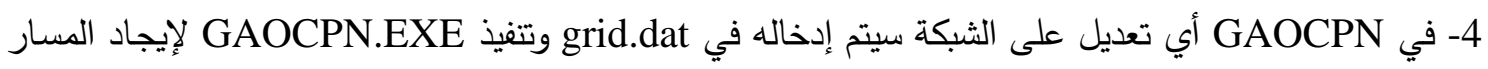
الحرج بينما في Win QSB وغيره يجب إعادة تصميم دالة الهدف والقيود ثم أيجاد المسار الحرج. 5- نظرا للنقطتين (3) و (4) فان GAOCPN يحقق مسألة الوقت الحقيقي Real Time بينما البرامج الجاهزة تحتاج إلى تصميم أنموذج خاص بكل مسألة وهذا يستغرق وقتاً لتمثيل الثبكة على هيئة نموذج بشكل صحيح

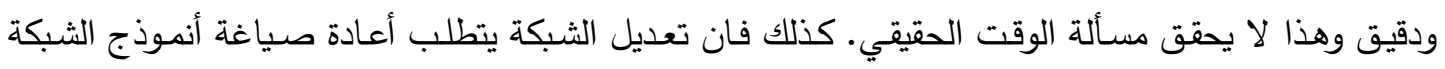
وتعديل دالة هدفه وقيوده.

6- هناك عدة نماذج رياضية طورت لجدولة المشاريع في الثلاثين سنة الأخيرة واستخدمت هذه النماذج (البرمجة

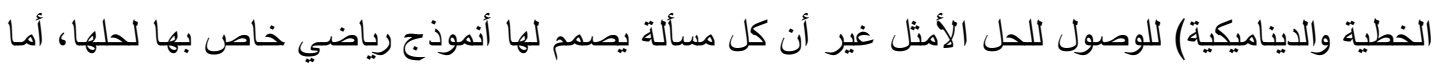
إمكانيات GA فهي خوارزمية عامة لجميع المسائل. 7- نماذج المحاكاة كال GA فهي خوارزمية عامـة لكل مسائل الأمثلية وتعطي زاويـة جديدة للرؤيا مخالف لحقل فئل

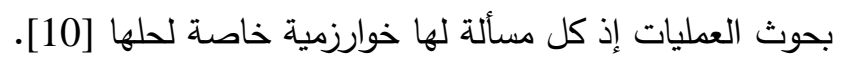

GATSP مع الخوارزمية الجينية للبائع المتجول GAOCPN 2-7 مقارنة ترميز كروموسومات GAOCPN أكثر تعقيدا مقارنة بالبائع المتجول للأسباب التالية: 1- في GAOCPN فان جينات الكروموسوم تمثل نشاط بينما في (GATSP) فان كل جين يمثل مدينة.

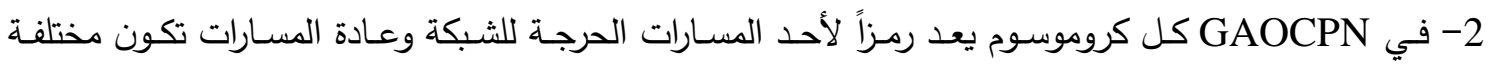
الأطوال لأن عدد العقد في كل مسار ليس ثابتا، بينما في (GATSP) فان الكروموسومات جميعها ثابتـة الطول لأن البائع المتجول يزور جميع المدن دون استثناء ويختلف كروموسوم عن آخر باختلاف ترتيب المدن التي سيزورها البائع. 3- في شبكة المشاريع لا يشترط ربط النشاط بجميع الأنشطة بينما شبكة البائع المتجول فكل مدينة مرتبطة بجميع

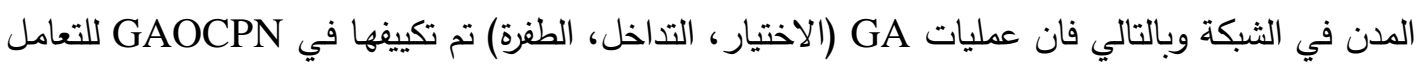
مع مسارات حقيقية فقط بينما عمليات GA في (GATSP) ستولد دائما مسارات مطابقة لشبكة (TSP). 
4- كروموسومات GAOCPN لا تمثل المسار بشكل مباشر بل نستخدم الترميز لإنشاء مسار إذ يتم ترميز الجينات على أساس الأولوية والتي تهتم بوزن للجينات (العقد) أما كروموسومات (GATSP) فإنها تمثل المسار نفسه. 5- توزيع العقد بشكل عشوائي بالنسبة لا.GAOCPN سوف لا يطابق أي مسار • بينما (GATSP) سيطابق المسألة. 6- هناك دليلان لكون GAOCPN نوع (NP Hard Problem) أولهما أن نتائج البحوث لحساب أفضل مسار

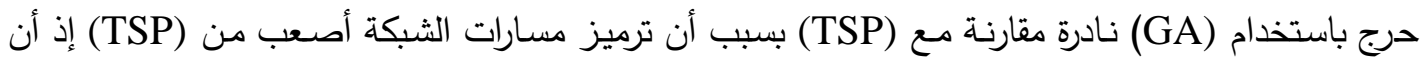

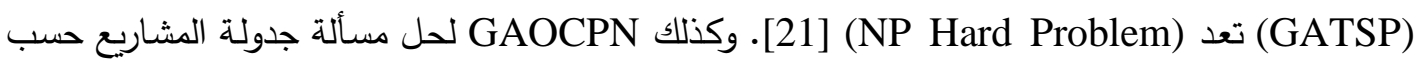

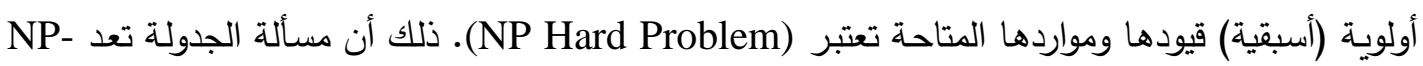

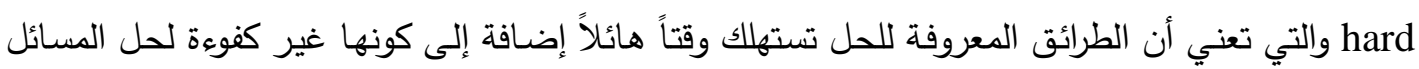

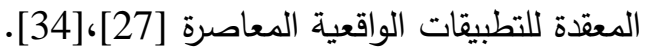

\section{8- وصف وتصميم خوارزمية GAOCPN}

أساس عمل الخوارزمية هو إعطاء قيم أولية وتعد قيماً أولوية لاختيار العقدة مما سيسمح للخوارزمية بتوليد مسار حقيقي قد يرشح لاحقاً ليكون هو المسار الحرج الأمثل [12]،24]. الشكل (4) يوضح الكية المخطط الانسيابي للخوارزمية المقترحة GAOCPN.

\section{1-8 وصف هيكل بيانات الكروموسوم (مجموعة القرار)}

أن الكروموسوم يمثل أحد الحلول الموجودة ضمن مجال البحث وبالتالي فهو أساس مجموعة القرار لكونه

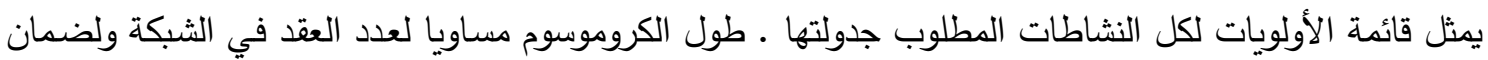

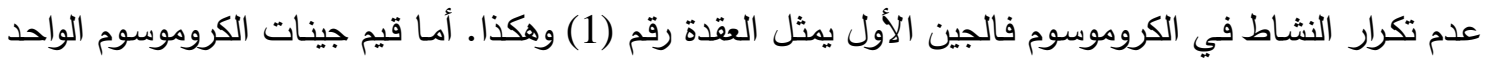

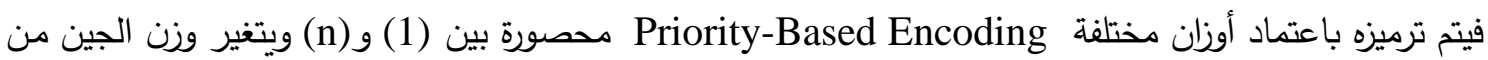

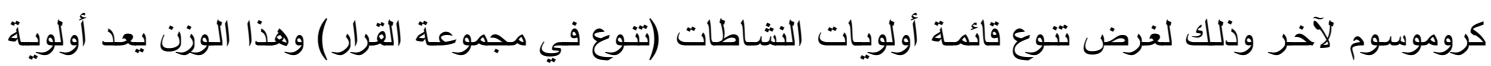

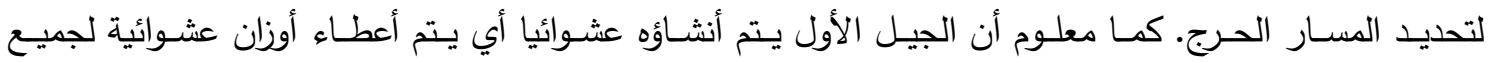

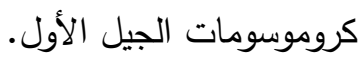

2-8 وصف هيكل بيانات شبكة المشروع (مجموعة الجدولة)

وذلك بمسح الثبكة من اليسار وابتداءً بعقدة البداية (1) إذ يتم خزن العقدة والعقد المرتبطة بها في صف وخزن قيمها في الصف التالي وتستمر عملية الخزن تباعا وحسب تسلسل عقد الثبكة وباتجاه اليمين انتهاءً بعقدة النهاية (n). لاحظ الجدول (7)، إذ تعد العقدة 20 نشاطاً أخيراً لجدولة المشروع لكونها غير مرتبطة بأخرى. 


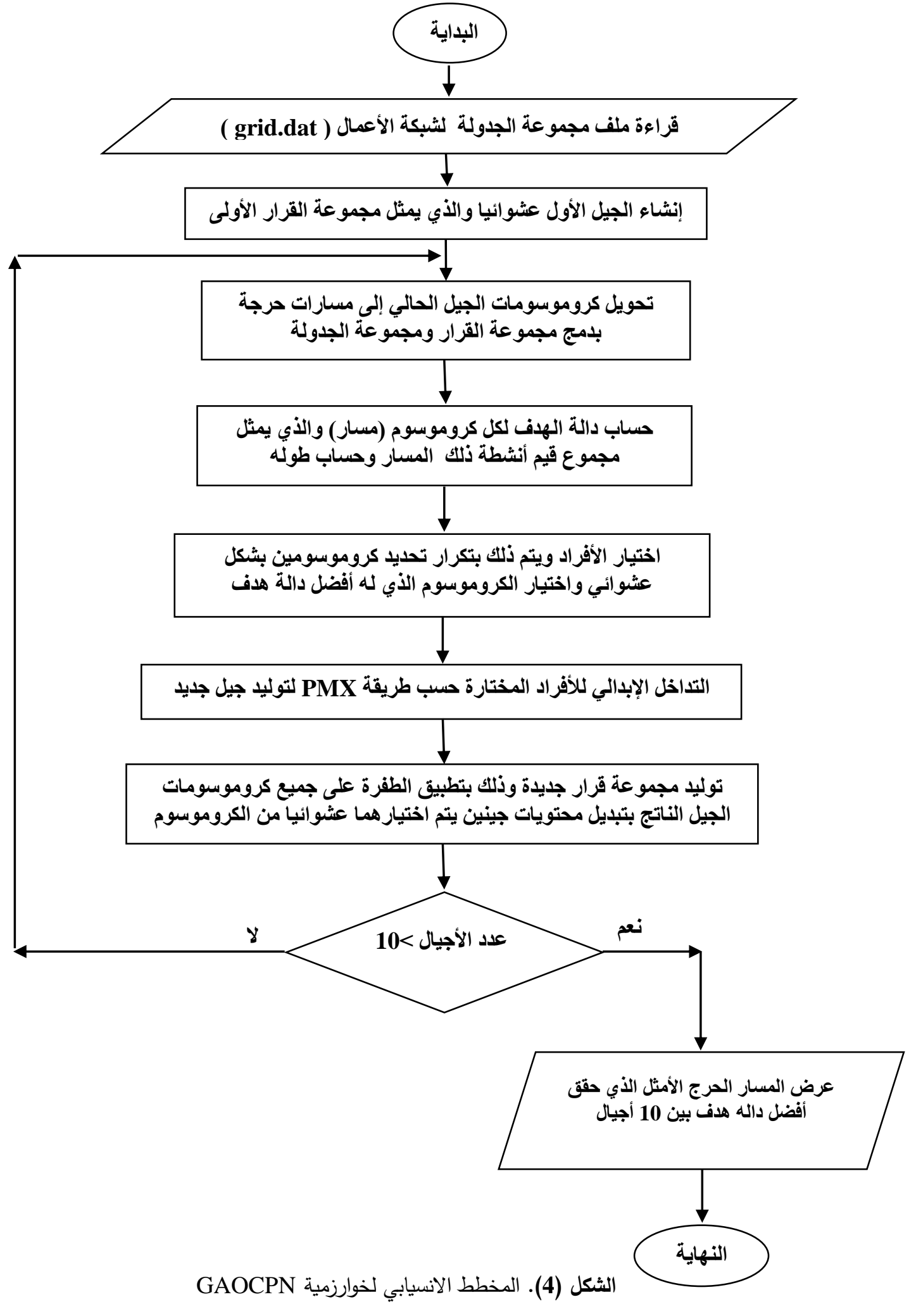

3-8 دمج مجموعة القرار ومجموعة الجدولة لتحويل الكروموسوم إلى مسار حرج حقيقي

كل كروموسوم يمثل مساراً حرجاً واقعياً ولاستتباط المسار منه نتبع الخطوات التالية [11]: 1- لجميع الثبكات أن أول نثاط فيها هو العقدة (1) أي أن جميع المسارات الحرجة تبدأ بالجين الأول فيكون: 
Current_node $=1$, Path $=1$, Path_length $=1$

2- تطبيق احد الحالات الثلاثة التالية:

الحالـة الأولىى: في حالة كون العقدة الحالية Current_node مرتبطة بعقدة واحدة (أي لها تفرع واحد) ولتكن

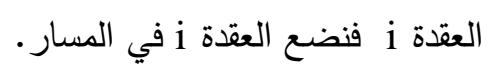

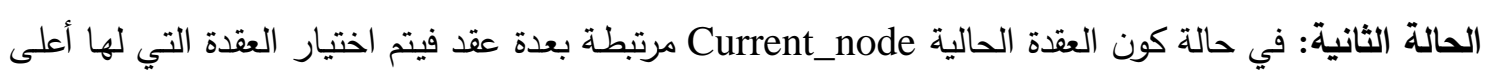

وزن حسب مجموعة قرار الكروموسوم ولنفترض هي العقدة i ووضعها ضمن المسار .

في حالة تحقق الحالة الأولى أو الثانية نفذ التالي:

Current_node $=$ i, Path $=$ Path $\rightarrow$ i, Path_length $=$ Path_length +1 , Goto step 2

الحالة الثالثة : أما في حالة عدم تحقق الحالتين الأولى والثانية فهذا يعني أن العقدة الحالية Current_node

غير مرتبطة بأي عقدة أي أنها آخر عقدة بالثبكة، عندها تم إيجاد المسار الحرج كاملا (Path)

حسب قانون القرار وحسـاب عدد عقد ذلك المسار Path_length ثم حسـاب الهدف للمسار

.Path

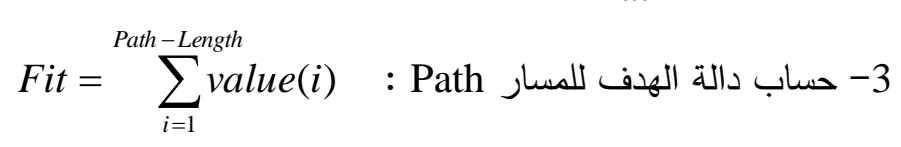

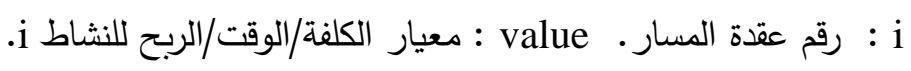

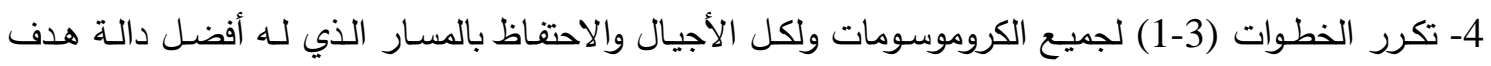

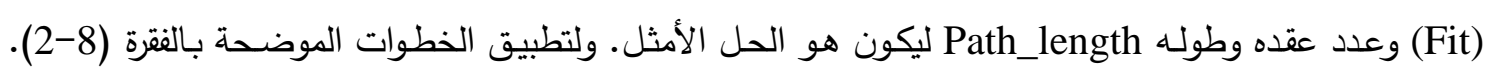
الجدول (8) يمثل أحد الكروموسومات.

الجدول (8). كروموسوم يمثل مجموعة القرار لمسار حقيقي في شبكة جسر الصرافية

\begin{tabular}{|l|l|l|l|l|l|l|l|l|l|l|l|l|l|l|l|l|l|l|l|l|}
\hline 20 & 19 & 18 & 17 & 16 & 15 & 14 & 13 & 12 & 11 & 10 & 9 & 8 & 7 & 6 & 5 & 4 & 3 & 2 & 1 & \begin{tabular}{c} 
رالعقدة \\
\hline
\end{tabular} \\
\hline 14 & 19 & 17 & 6 & 16 & 12 & 9 & 15 & 13 & 4 & 20 & 2 & 11 & 8 & 18 & 3 & 10 & 1 & 7 & 5 & الوزن \\
\hline
\end{tabular}

ولتحديد المسار الحرج لمجموعة القرار للكروموسوم أعلاه, وحسب الخطوة 1 نبدأ من الجين الأول لكونـه

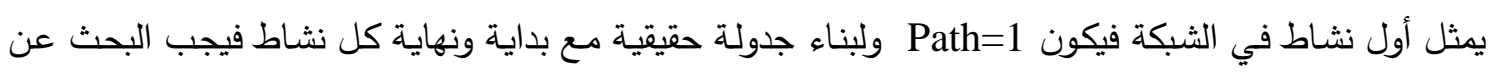
النشاط التالي الذي ممكن تأديته بدون انتهاك لقيود الثبكة وبالرجوع إلى المـلف (grid.dat) الذي يمثل مجموعة

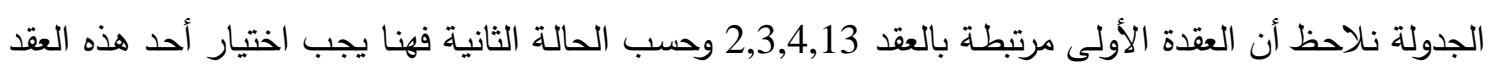

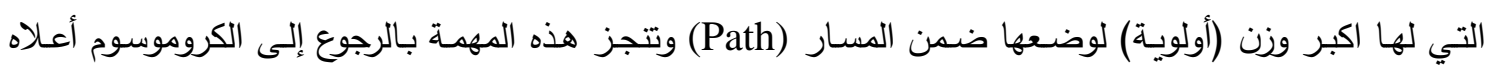

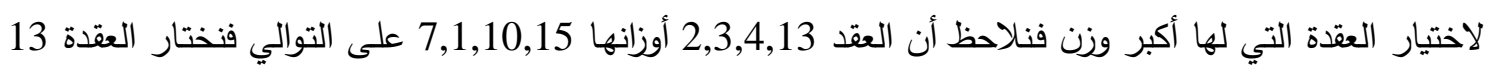

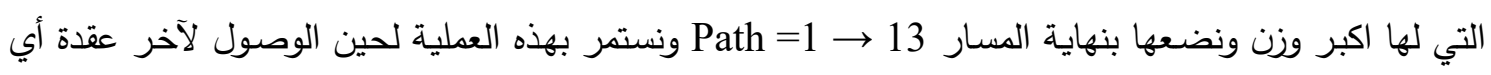
لعقدة خالية من التفرعات. وبالرجوع للملف (grid.dat) نلاحظ أن العقدة (13) مرتبطة بعقدة واحدة وهي العقدة

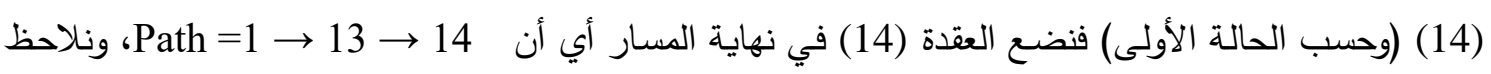
أيضا أن العقدة (14) مرتبطة بعقدة واحدة وهي (20)، أيضا تضاف مباشرة لنهاية المسار فيكون المسار : Path

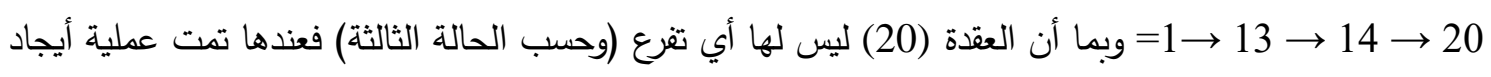
مسار واقعي ذي طول 4 عقد (3 خطوات) والذي قيمة دالة الهدف لـه هو 42 يوماً. إذ أن مجموع قيم أنثطة المسار هو Fit = D+W+X أي أن Fit=30+10+2 = 42 ( 
GAOCPN وصف برنامج

أن GAOCPN.EXE هو صندوق اسود لجدولة المشاريع أو تحسين الحلول السابقة للجدولة باختيار

أمثلها حسب معيار الوقت،الكلفة أو الربح مع التقيد بهيكل وقيود الثبكة إذ يتم خزن الثبكة بملف مجموعة الجدولة (grid.dat) ومراحل تتفيذ النظام كالآتي:

1- مرحلة تهيئة عوامل الخوارزمية الجينية المقترحة: والمتمثلة بالجدول (9).

الجدول (9). عوامل الخوارزمية الجينية GAOCPN

\begin{tabular}{|l|l|}
\hline Population Size ( pop_size ) & 10 \\
\hline No Of Generations & 10 \\
\hline Selection Method & Binary select method \\
\hline Crossover Method & Partially Mapped Crossover PMX \\
\hline Mutation Method & simple swapping \\
\hline Chromosome Size & No. of nodes in project network = N \\
\hline
\end{tabular}

2- مرحلـة الإدخـال: على المستخدم (مدير المشـروع) تزويـــ النظـام بملف (grid.dat) الذي يحـوي مجموعـة الجدولة (الأنشطة وقيمها) ثم يختار دالة الهدف (أقل كلفة أو وقت، أعلى ربح) وذلكالك بإدخال الرقم المقابل لدالة الهدف والبرنامج بمثابة صندوق اسود لكونه حلقة وصل لقراءة البيانات المدخلة ويقيم المسارات حسب لتبات الدالة المختارة.

3- مرحلة عرض النتائج: سيتم أظهار أفضل مسار تم إيجاده وطوله في كل جيل.

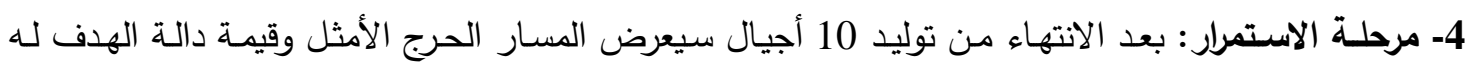
وطوله وعدد عقده ثم تظهر عبارة continue(Y/N)? فإذا رغب المستخدم بالاستمرار بتوليد (10) أجيال

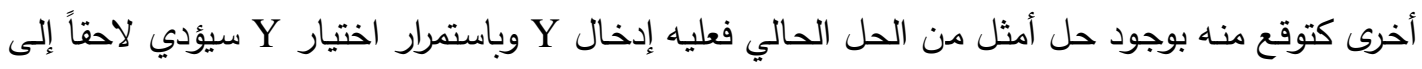
استقرار الحل ولا يعطي التنفيذ أي تحسن للحل فهنا ليس هناك جدوى من الاستمرار في التنفيذ وعندها ننصح

المستخدم بإدخال N لإيقاف التتفيذ واعتماد المسار الذي يظهره البرنامج بكونه أمثل مسار حرج للثشبكة. ففي الثبكة الموضحة بالثكل (1) تحوي على 13 مرحلة و29 نشاط و20 عقدة تم الوصول إلى الحل

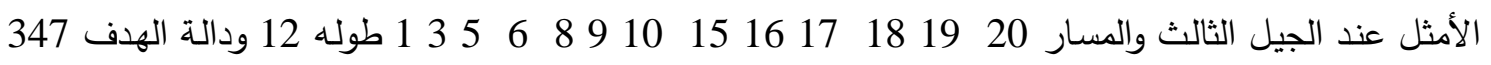
يوماً. وخوارزميات البرامج الفرعية لبرنامج GAOCPN.CPP موضحة كالآتي:

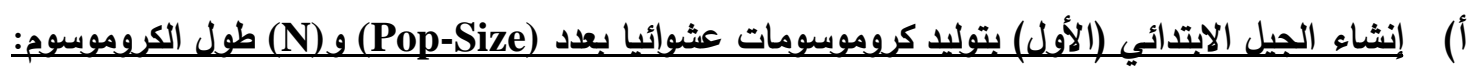
1) لجميع قيم i حيث i

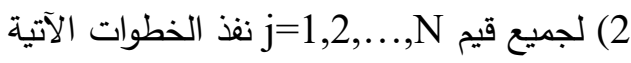

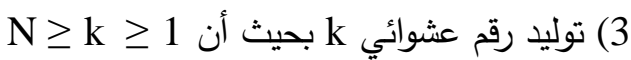
4) في حالـة تكرار الجين k في الكروموسوم i أذهب إلى الخى الخطوة (3) وإلا ضـع الجين k في الموقع j مـن

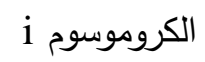
5) أذهب إلى الخطوة (2) 6) في حالة تكرار الكروموسوم i في الجيل الأول أذهب إلى الخطوة (2) 
(1) (1) (1) (1) (إذب )

ب) أيجاد المسار المطابق للكروموسوم وحساب دالة الهـف:

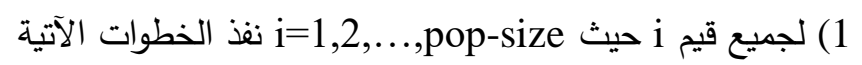

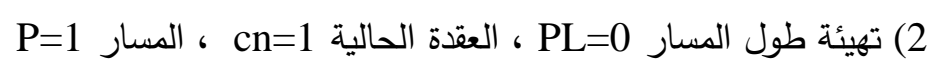

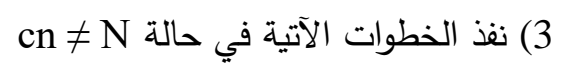
$\mathrm{PL}=\mathrm{PL}+1$ (4

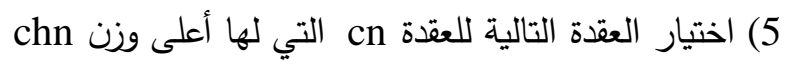

$\mathrm{cn}=\operatorname{chn}, \mathrm{P}=\mathrm{P} \rightarrow \mathrm{cn}(6$

(3) (3) أذهب إلى الخطوة (1) (1)

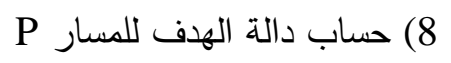

9) أذهب إلى الخطوة (1) (1)

ج) انتقاء الآباء:

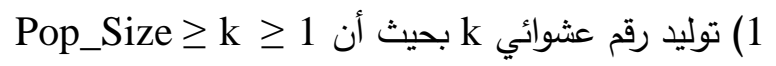

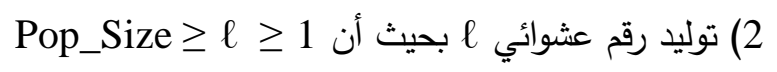

3) أذا كانت

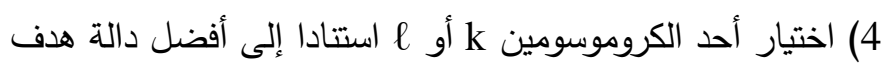

د) التداخل الإبدالي لتوليد الأبناء:

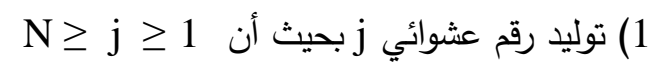

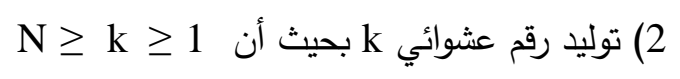

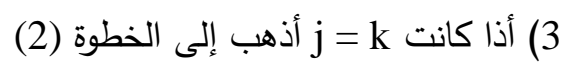

4) إجراء عملية التداخل الإبدالي للجينات الواقعة بين j و الكل كروموسومين متتاليين من الآباء

هـ) الطفرة:

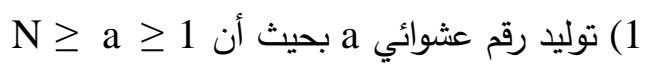

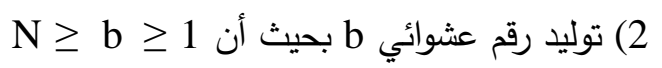

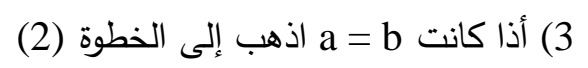

4) أجراء عملية الطفرة بالمبادلة بين الجين a و b لكروموسومات الأبناء

5- آلية عمل GAOCPN: بالنسبة لآلية التنفيذ فان نتيجة ووقت التنفيذ لأول خمسة أجيال كانت كالآتي:

حيث أن صيغة الوقت Hour: Minute: Second. Hundredths of seconds

\section{The current time is: $\mathbf{1 0 : 2 6 : 3 5 . 5 0}$}

.. GENETIC ALG. FOR PATH SCHEDULING

The Fitness Function according to :

1- MAXIMIZATION (Profit or days).

2- MINIMIZATION (Cost or Time).

Select (1 or 2): 1 


\begin{tabular}{|c|c|c|c|c|}
\hline \multirow{2}{*}{ تسلسل } & \multirow{2}{*}{ الكروموسوم } & أفضل كروموسو بالجيل الذي يحقق أفضل مسار & \multirow{2}{*}{ قليمة دالة } & \multirow{2}{*}{ المسار } \\
\hline & & أفضل مسار بالجيل & & \\
\hline \multirow{2}{*}{1} & \multirow{2}{*}{1} & 8132010142561716914111219718153 & \multirow{2}{*}{283} & \multirow{2}{*}{11} \\
\hline & & 133588910151617181920 & & \\
\hline \multirow{2}{*}{2} & \multirow{2}{*}{6} & 1613201019172564129171114183815 & \multirow{2}{*}{312} & \multirow{2}{*}{10} \\
\hline & & $13350688911121314 \quad 20$ & & \\
\hline \multirow{2}{*}{3} & \multirow{2}{*}{9} & 4141651015116131789220121971813 & \multirow{2}{*}{347} & \multirow{2}{*}{12} \\
\hline & & $135668910 \quad 151617 \quad 181920$ & & \\
\hline \multirow{2}{*}{4} & \multirow{2}{*}{10} & 1014128181316752011317162941519 & \multirow{2}{*}{285} & \multirow{2}{*}{9} \\
\hline & & $123568911 \quad 1420$ & & \\
\hline \multirow[t]{2}{*}{5} & \multirow{2}{*}{7} & 2916151310751119312142018176184 & \multirow{2}{*}{346} & \multirow{2}{*}{11} \\
\hline & & $\begin{array}{lllllllllllll}1 & 3 & 5 & 6 & 8 & 9 & 10 & 15 & 17 & 18 & 19 & 20 & \\
\end{array}$ & & \\
\hline
\end{tabular}

THE OPTIMAL Path ACCORDING TO Max. (Profit/days) is: $\begin{array}{llllllll}1 & 3 & 5 & 6 & 8 & 9 & 10 & 15\end{array}$ $\begin{array}{lllllll}16 & 1 & 7 & 18 & 19 & 20\end{array}$

Fitness $=347$ Days.

No. of Steps $=12$

The current time is: 10:26:35.52

Continue $(\mathrm{y} / \mathrm{n}) ?: \mathbf{n}$

...... PRESS ANY KEY TO EXIT ......

6- تقييم تصميم وأداء GAOCPN:

1- إن الملف المقترح من قبل الباحثين لهيكل بيانات مجموعة الجدولة، الذي يخص جسر الصرافية المثلة بعشرين عقدة ذي حجم 277 بايت وبالمقابل فان تمثيلها كمصفوفة لكانت أبعادها 20*20*4 (حجم العدد) أي 1600 بايت وبهذا يتضح أن صيغة هيكل ملف مجموعة الجدولة أكفأ من المصفوفة. 2- يتميز تصميم GAOCPN.CPP بجعل برنـامج فرعي خـاص بكل خطوة مـن خطوات الخوارزميـة الجينيـة وبالتالي سهولة تطويره واستحداثه من قبل المبرمج مستقبلاً.

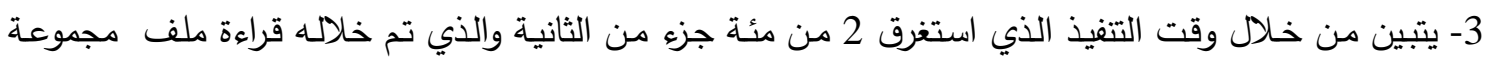

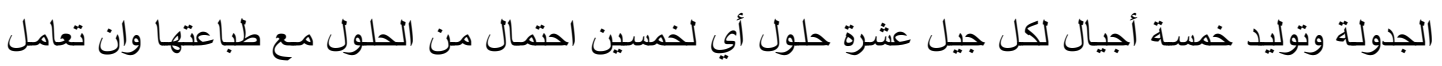
وحدة المعالجة المركزية CPU مع أجهزة الإدخال والإخراج استغرق الوقت الأكبر من وقت التولت التنفيذ.

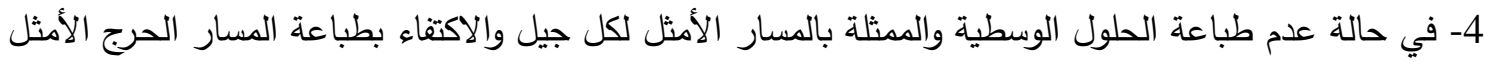

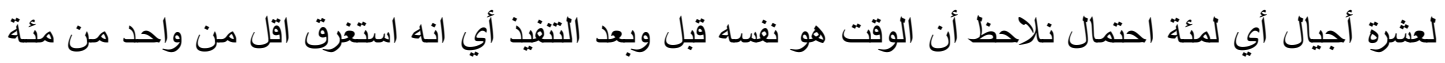
جزء من الثانية.

5- خطوات التتفيذ تحقق ميزات الخوارزميـة الجينية من ناحية الرصانة Robustness كأن يكون الحل الأمثل

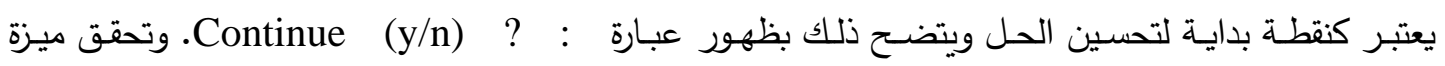

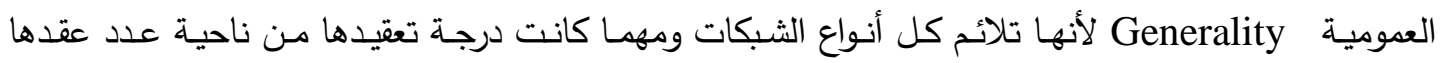
ومساراتها. وكذلك الدقة Accuracy والقدرة على إيجاد حل امثل ودقيق وهذا لا يتطلب خبرة في الأمثلية وبحوث العمليات، وأخيرا يمتاز GAOCPN بسهولة تتفيذه ولا يتطلب أي مهارة أو خبرة لتتفيذه. 
1- أظهرت النتائج أن GAOCPN أكثر ملائمة لمسائل الجدولة المعاصرة لكونه مرناً تمامـا بما يخص التعديل على هيكل الثبكة ودقيق وكفوء لحل أحد المسائل التي تخص جدولة المشاريع فهي أداة مهـة وذات قيمـة

$$
\text { عظيمة لمدراء المشاريع لإدارة مشاريعهم حاسوبيا. }
$$

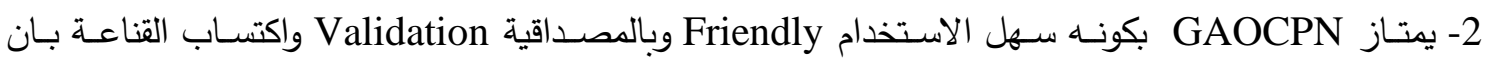

$$
\text { النموذج يعمل واقعياً وبنجاح. }
$$

GAOCPN -3 أعطت فرصة أكبر لاستخدام مسائل الأمثلية كمرشد (دليل) لمدير المشروع لاتخاذ القرار الصائب

$$
\text { الذي يمثل أعلى ملائمة في مجال البحث. }
$$

4- إن أسلوب دمج مجموعة القرار ومجموعة الجدولة سرع عملية الاقتراب من الحل والوصول للحل لأنها تغطي كل المسارات الحرجة (فضاء البحث) ومن ضمنها المسار الحرج الأمثل.

5- استخدام تقنيات البحث الذكية GA GA لحل مسائل بحوث العمليات وبالأخص ومنها (البرمجة الخطية). 6- بالنسبة لطريقة البرمجة الديناميكية كان الحد الأعلى لانجاز مشروع جسر الصرافية حسب الطريقة المباشرة هو

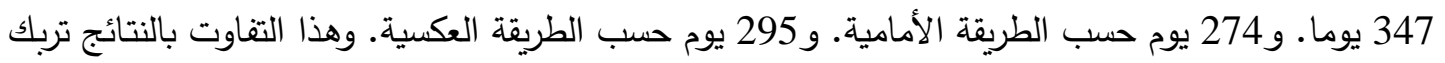

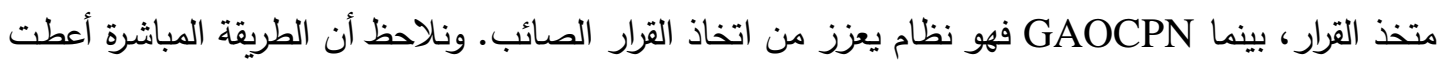

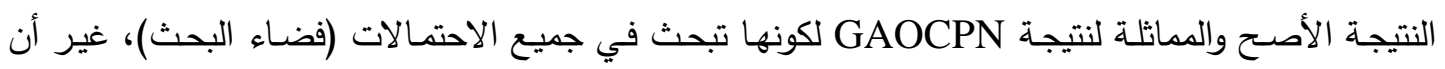
GAOCPN 7- تفوق الخوارزميـة الجينيـة المقترحة على بحوث العمليات فالخوارزميـة الجينيـة لا تعتمد على معرفتنـا بطرق

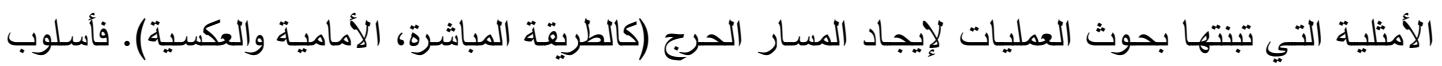
الخوارزمية الجينية مستقل ومختلف تماما وكليا عن هذه الطرق التقليدية بوصفه احد التقنيات الذكائية.

1- نؤكد ونوصسي باستخدام التقنيات الذكائية كالخوارزمية الجينية (GA) والمنطق المضبب (Fuzzy Logic) والثبكات العصبية (Neural Networks) بوصفها أساليب حديثة تضاف لمجال بحوث العمليات ومقارنـة نتائجها مع نتائج الطرق التقليدية لحل مسائل بحوث العمليات ودراستها وتحليلها علميا واستتباط الأكفأ منها لاعتمادها عمليا في مجال بحوث العمليات. 2- أن أي تغيير في خطة المشروع يتطلب تعديل شبكة المشروع وذلك بتعديل ملف (grid.dat) ثم أعادة تتفيذ (GAOCPN.EXE)

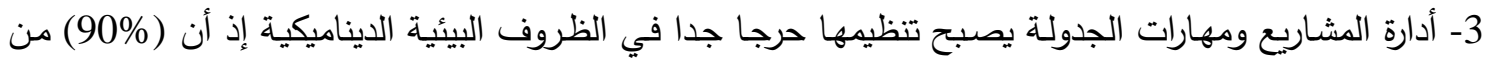
المشاريع تعد بيئة مشاريع متعددة (multi projects) وتتفذ بشكل عدة مشاريع فرعية وهذا النوع من المشاريع

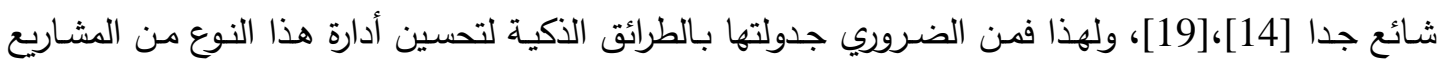

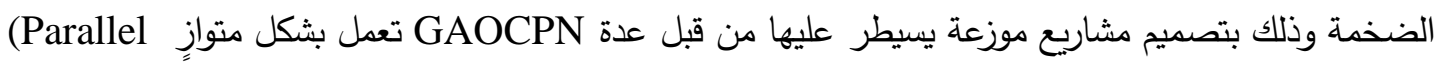

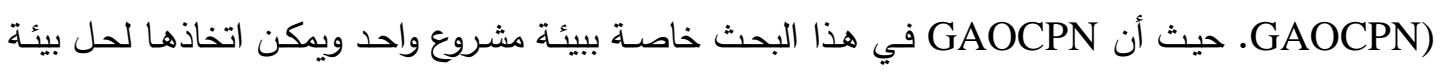
المشاريع مثل المشاريع النووية، صناعة الصواريخ العابرة للقارات، مكوك الفضاء، صناعة الطائرات. 


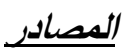

الراشدي، زيد محمد محمود(2009). " أسلوب بيرت باستخدام توزيع ثثائي القوة في تخطيط ومتابعة

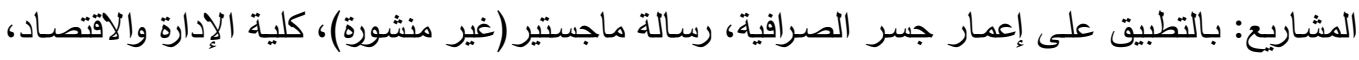

جامعة الموصل.

[2] Abraham A., Jain, L. and Goldberg R., (2005). "Evolutionary multiobjective optimization: Theoretical Advances and Applications", Spring-Verlang, USA.

[3] Ackley, A., Baker, J., and Lowe, S. (2007). "Top ten CPM scheduling mistakes."Rail Conference 2007 Proceedings, June, 2007, Toronto, On.

[4] Alba, E. and Chicano, J.F., (2007), "Software project management with GAs", Information sciences, Vol.177, Issue 11, pp.2380-2401.

[5] Deiranlou, M; Jolai, F. (2009), "A New Efficient Genetic Algorithm for project scheduling under resource constraints", World Applied Sciences Journal 7(8), pp.987-997.

[6] Demeulemeester, E.L. and Herroelen, W.S., (2002), "Project Scheduling: A Research Handbook", Kluwer Academic Publishers, USA.

[7] Eiselt, H.A. and Sandblom, C.L. (2010), " Operations Reseearch A Model-Based Approach", Springer -Verlag Berlin Heidelbrg, Germany.

[8] Elbeltagi, E.; Elkassas, E.; Abdel Rasheed, I. and AL-Tawil, S., (2007), " Scheduling and cost optimization of repetitive projects using genetic algorithms", ICCTA, pp.319-327.

[9] Eshtehardian, E., Afshr, A., and Abbasnia, R. (2008). "Time-cost optimization: using GA and fuzzy sets theory for uncertainties in cost." Construction Management and Economics, 26, 679-691.

[10] Gonçalves, J. F. (2002), "Mendes, J.J.M., Resende, M. G. C.: A hybrid genetic algorithm for the job shop scheduling problem", AT\&T Labs Research Technical Report TD-5EAL6J, AT\&T Labs Research.

[11] Hartmann, S., (1997), "A Competitive Genetic Algorithm for ResourceConstrained Project Scheduling", No.451,Manuskipte aus den instituten fur Betriebswiertsch- aftslehre der universitat Kiel.

[12] Hartmann,S.(2002), "A Self-Adapting Genetic Algorithm for Project Scheduling under Resource Constraints", Naval Research Logistics,49,pp.433-448.

[13] Hutchings, J.E., (2004), "Project Scheduling Handbook", Marcel Dekker, Inc.. USA.

[14] J.F. Gonçalves, J.J. de Magalhaes Mendes, M.G.C. Resende, A Genetic Algorithm for the Resource Constrained Multi-Project Scheduling Problem, AT\&T Labs Technical Report TD-668LM4, (2004).

[15] Justesen,P.D.(2009),"Multi-Objective Optimization using Evolutionary Algorithm", University of Aarhus, Denmark.

[16] Kam, C.(2005)," Dynamic Decision Breakdown Structure Ontology, Methodology, and Framework for Information Management in Support of 
Decision-Enabling Tasks in the Building Industry", Civil and Environmental Engineering of Stanford University (2005).

[17] Karova, M.; Petkova, J. and Smarkov, V., (2008), "A Genetic Algorithm for Project Planning Problem", International Scientific Conference Computer Science, pp.647-651.

[18] Khalaf, W.S.; June, L.W., (2009), "A linear programming approach for the project controlling", Research Journal of Applied Sciences, 4(5), pp.202-212.

[19] Kilic, M., (2003), "Multiobjective Genetic Algorithm Approaches to Project Scheduling Under Risk", MS.C Thesis, Graduate School of Engineeing and Natural Sciences, Sabanic University .

[20] Lew, A. and Mauch, H., (2007), "Dynamic Programming A Computational Tool",Springer__ Verlag Berlin Heidelberg. New York ,USA .

[21] Li, Y.; He, R.and Guo, Y. (2006), "Faster Genetic Algorithm for Network Paths", The Sixth International Symposium on Operations Research and its Applictions (ISORA), china PP.380 -389.

[22] Lin, L., and Gen, M., (2009), "Priority -Based Genetic Algorithm for Shortest Path Routing Problem in OSPF", Intelligent and Evolutinary Systems, SCI 187-, PP.91 -103.

[23] Menesi, W., (2010), "Construction Scheduling Using Critical Path Analysis with Separate Time Segments, "Ph.D Thesis, University of Waterloo, Waterloo, Canada.

[24] Mitsuo Gen and Lin Lin, (2005), "Multi-objective hybrid genetic algorithm for bicriteria network design problem", Graduate School of Information, Production \& Systems, Waseda University, Wakamatsu-ku, Complexity International Vol.11, Kitakyushu 808-0135, JAPAN,pp.73-83.

[25] Raviravindran, A., (2009), "Operations Research Applictions", CRC Press, Taylor \& Francis Group, LLC, USA .

[26] Roy, G.L AND Stelth, P., (2009), "Projeots`Analysis Through CPM (Critical Path Method)", School of Doctoral Studies (European Union) Journal, No.1, PP.10-51.

[27] R. Kolisch, R. Padman, An integrated survey of project deterministic scheduling, International Journal of Management Science 29(3) (2001) 249-272.

[28] Shouman, M.A., Ibrahim, M.S; Khater, and Forgani, A.A., (2006), "Genetic Algorithm Constraint Project Scheduling", Alexandria Engineering Journal, V.45, No.3, PP.289-298.

[29] Sivanandam, S.N. and Deepa, S.N., (2008),"Introduction to Genetic Algorithms", Springer-Verlag Berlin Heidelberg, New York, USA.

[30] Sniedovich, M., (2011), "Dynamic Programming Foundation and Principles", Second Edition, CRC Pres, Taylor and Francis Group, LLC, USA .

[31] Srivastava, P.R. and Kim, T-H, (2009), "Application of Genetic Algorithm in Software Testing", International Journal of Software Engineering and its Applications, Vol. 3, No.4, PP.87-95. 
[32] T.R. Browning, (2001), "Applying the Design Structure Matrix to System Decomposition and Integration Problems: A Review and New Directions", IEEE Transactions on Engineering Management, 48(3), 292-306.

[33] Valls, V., Ballestín, F., Quintanilla, S., (2007), "A Hybrid Genetic Algorithm for the Resource-Constrained Project Scheduling Problem, European Journal of Operational Research.

[34] Woolf, M. (2008). "When is the critical path not the most critical path?" AACE International Transactions, PS.07.1-PS.07.9.

[35] Zahraie, B. and Tavakolan, M. (2009). "Stochastic time-cost-resource utilization optimization using nondominated sorting genetic algorithm and discrete fuzzy sets." Journal of Construction Engineering and Management, ASCE, 135(11), $1162-1171$.

[36] Zhao, T.and Tseng, C-L, (2003), "A note on activity flouts in activity-on-arrow networks", Journal of the Operationl Reseach Society, 54, PP.1296 -1299.

[37] Zhegazy T., and Menesi W., (2010), "Critical Path Segments (CPS) scheduling technique", Journal of Construction Engineering and Management, ASCE, 136(10). 